TRANSACTIONS OF THE

AMERICAN MATHEMATICAL SOCIETY

Volume 349, Number 5, May 1997, Pages 2085-2113

S 0002-9947(97)01905-3

\title{
DUALITY OF RESTRICTION AND INDUCTION FOR $C^{*}$-COACTIONS
}

\author{
S. KALISZEWSKI, JOHN QUIGG, AND IAIN RAEBURN
}

\begin{abstract}
Consider a coaction $\delta$ of a locally compact group $G$ on a $C^{*}$ algebra $A$, and a closed normal subgroup $N$ of $G$. We prove, following results of Echterhoff for abelian $G$, that Mansfield's imprimitivity between $A \times_{\delta \mid} G / N$ and $A \times{ }_{\delta} G \times \times_{\hat{\delta}, r} N$ implements equivalences between Mansfield induction of representations from $A \times \times_{\delta \mid} G / N$ to $A \times_{\delta} G$ and restriction of representations from $A \times{ }_{\delta} G \times{ }_{\hat{\delta}, r} N$ to $A \times{ }_{\delta} G$, and between restriction of representations from $A \times{ }_{\delta} G$ to $A \times \times_{\delta \mid} G / N$ and Green induction of representations from $A \times{ }_{\delta} G$ to $A \times{ }_{\delta} G \times{ }_{\hat{\delta}, r} N$. This allows us to deduce properties of Mansfield induction from the known theory of ordinary crossed products.
\end{abstract}

\section{INTRODUCTION}

In applications of duality theory for crossed products one often has to know how common constructions such as induction and restriction of representations behave under duality. Thus, after piecemeal results by several authors, Echterhoff was led to prove that, for systems involving abelian groups, induction and restriction are dual to one another [Ech94a]. He later used these results to great effect in his analysis of crossed products with continuous trace [Ech].

To state Echterhoff's theorem precisely, we fix an action $\alpha$ of a locally compact abelian group $G$ on a $C^{*}$-algebra $A$, and a closed subgroup $N$ of $G$. Green's imprimitivity theorem gives an imprimitivity bimodule $X_{N}^{G}$ implementing a Morita equivalence between $A \times_{\alpha} N$ and the imprimitivity algebra $\left(A \otimes C_{0}(G / N)\right) \times_{\alpha \otimes \tau} G$; the latter algebra is naturally isomorphic to the crossed product $A \times{ }_{\alpha} G \times{ }_{\hat{\alpha}} N^{\perp}$ by the dual action $\hat{\alpha}$ of the subgroup $(G / N)^{\wedge}=N^{\perp}$ of $\hat{G}$. Let $\operatorname{Ind}_{N}^{G}: \operatorname{Rep} A \times_{\alpha}$ $N \rightarrow \operatorname{Rep} A \times{ }_{\alpha} G$ denote the map on equivalence classes of representations given by induction of representations in the sense of Green: if $\pi$ is a representation of $A \times{ }_{\alpha} N$ on $\mathcal{H}$, then Ind $\pi$ is by definition the natural left action on (a completion of) $X_{N}^{G} \otimes_{A \times{ }_{\alpha} N} \mathcal{H}$. Similarly, let $\operatorname{Res}_{G}^{N}: \operatorname{Rep} A \times{ }_{\alpha} G \rightarrow \operatorname{Rep} A \times{ }_{\alpha} N$ denote the map given by restricting a covariant representation $(\pi, U)$ of $(A, G, \alpha)$ to the representation $\left(\pi,\left.U\right|_{N}\right)$ of $(A, N, \alpha)$. Then Echterhoff's theorem says that we have commutative

Received by the editors December 11, 1995.

1991 Mathematics Subject Classification. Primary 46L55.

This research was partially supported by the National Science Foundation under Grant No. DMS9401253, and by the Australian Research Council. 
diagrams

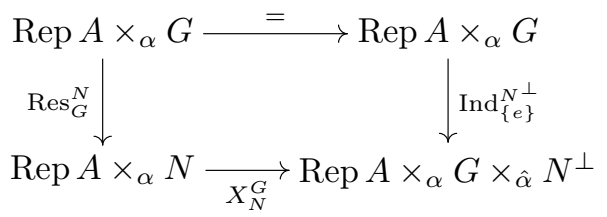

and

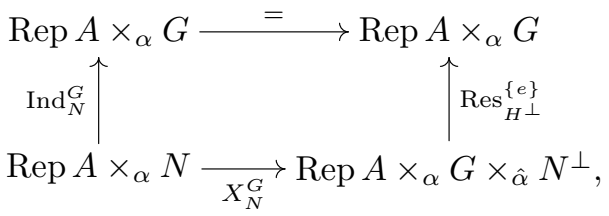

in which the horizontal arrows on the bottom are the bijections induced by the imprimitivity bimodule $X_{N}^{G}$.

As it stands, this theorem only makes sense for abelian groups, because otherwise there is no dual action. However, as Echterhoff himself observed, this is an obvious case of a theorem about abelian groups which should extend to an arbitrary locally compact group $G$ and a closed normal subgroup $N$, replacing dual actions by dual coactions. (Another such theorem is [OP86, Theorem 2.4], which was extended to non-abelian groups in [QR95, Theorem 4.4].) This project has been carried through in collaboration with Echterhoff; for this one has to relate induction and restriction for $A \times{ }_{\alpha} G$ to, respectively, the restriction and induction processes of Mansfield [Man91] for crossed products by coactions [EKR95].

Here we study the dual situation in which we start with a coaction $\delta$ of a locally compact group $G$ on $A$. Our main theorems give (in special cases) commutative diagrams

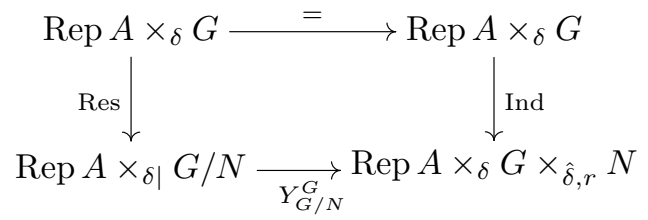

and

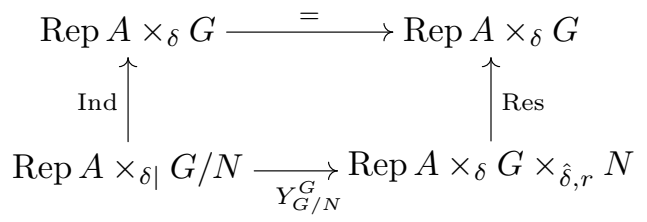

similar to (1.1) and (1.2). This time, the horizontal arrows are the bijections given by Mansfield's Morita equivalence of $A \times{ }_{\delta \mid} G / N$ with $A \times{ }_{\delta} G \times{ }_{\hat{\delta}, r} N$. These theorems are potentially very useful, since they reduce questions about Mansfield induction and restriction to questions about the much more familiar and manageable theory of Green [Gre78]. Indeed, in the special case $N=G$, diagrams (1.3) and (1.4) reduce to results used by Gootman and Lazar [GL89] to study induced ideals and the primitive ideal space of crossed products by coactions. 
We begin with a preliminary section in which we discuss our conventions about Hilbert modules, basic facts about induction of representations, and a version of Green's imprimitivity theorem for reduced crossed products. This last is necessary because the version of Mansfield imprimitivity for non-amenable groups developed in $[\mathrm{KQ}]$ used the reduced crossed product by the dual action, and we want to use this to avoid unnecessary assumptions about amenability. We stress, however, that for amenable groups, the only part of [KQ] needed here is the extension of Mansfield's theorem to full coactions and twisted systems. A special case of this extension is in [PR89], and the general case is hinted at in [ER96].

Our first main results in Section 3 concern the "Res-Ind" diagram (1.3). The result is more general than that stated above in three respects. First, we consider two normal subgroups $N \subset H \subset G$. Second, we consider also the twisted crossed products of [PR94], which introduces another normal subgroup $K$ containing $H$; our strategy, both here and in Section 4, is to prove the untwisted version, and then check that the constructions respect the twists. Third, we prove that the diagram commutes in a very strong sense: all the maps in the diagram are implemented by Hilbert modules, so their compositions are given by tensor products of modules, and we prove that the tensor products corresponding to the two alternative routes are naturally isomorphic. This not only gives a stronger theorem than the corresponding result of [Ech94a], but also a distinctly shorter proof. Thus we can claim that this is another example of a theorem about abelian groups which becomes much cleaner when seen from the point of view of coactions.

In Section 4 we turn to the "Ind-Res" diagram (1.4). As in Section 3, our main results are much more general than stated above. The overall pattern of this section is like that of Section 3, but this time passing to twisted crossed products is harder. In Echterhoff's paper, the second diagram (1.2) amounts to the induction in stages of Green [Gre78]. Mansfield did not prove such a theorem for his induction process, so we are forced to do it here (Corollary 4.2).

In Section 5 we give some applications of our theorems. We prove that restriction and induction are compatible with Morita equivalence in general, and in particular with the stabilization trick of [ER96], which allows us to replace twisted crossed products by ordinary ones. (This result for abelian groups was a fundamental tool in [Ech].) Along the way we deduce, from the corresponding properties of Ind and Res for crossed products by actions, that both Res and Ind are respected by Morita equivalence of coactions. We feel that this is a good illustration of how our results might be used to deduce information about coactions from known properties of actions.

Finally, in the last section, we use our main theorems to study the maps Res, Ind, Ex and Sub on ideals of crossed products by coactions, obtaining generalizations to the case of nonamenable $G$ of many of the results in [GL89, §3]. Here we see the power not only of Res-Ind (and Sub-Ex) duality, but also that of the Hilbert module techniques, since our maps on spaces of representations automatically give rise, via the Rieffel correspondence, to maps on spaces of ideals.

This research was carried out while the second author was visiting the University of Newcastle in 1994 and 1995, and while the first author was visiting Arizona State University in 1995. The various visitors are grateful to their respective hosts for their hospitality. The authors thank the referee for some helpful suggestions, particularly concerning the proof of Corollary 4.3 . 


\section{Preliminaries}

Throughout, $G$ will be a locally compact group with modular function $\Delta_{G}$. We use left Haar measure. The group $C^{*}$-algebra of $G$ is denoted $C^{*}(G)$; a subscript $r$, as in $C_{r}^{*}(G)$ or $B \times{ }_{r} G$, always indicates a reduced object. Nondegenerate homomorphisms of $C^{*}$-algebras extend to homomorphisms of their multiplier algebras, and this will be done implicitly.

Coactions and Imprimitivity. We use the conventions of [KQ], [Qui95], [QR95, Section 7], and [Rae92], although the latter uses maximal tensor products. Our coactions use minimal tensor products, are injective, and are full, i.e., use $C^{*}(G)$. Let $(A, G, \delta)$ be a coaction. We let $\left(A \times G, j_{A}, j_{G}\right)$ denote the crossed product, $\hat{\delta}$ the dual action of $G$ on $A \times G$, and $\delta^{\mathrm{n}}$ the normalization of $\delta$ [Qui95, Definition 2.4]. If $N$ is a closed normal subgroup of $G$, we let $\delta \mid$ denote the restricted coaction of $G / N$ on $A$ and $A \times G / N$ the restricted crossed product [Rae92, Example 2.3]. Moreover we let $\mu \mid$ denote the restriction to $C_{0}(G / N)$ of a nondegenerate homomorphism $\mu$ of $C_{0}(G)$.

In $[\mathrm{KQ}]$ the first two authors generalized Mansfield's imprimitivity machine [Man91]. When $(A, G, \delta)$ is a nondegenerate coaction and $N$ is a closed normal subgroup of $G$, there are dense $*$-subalgebras $\mathcal{D}$ and $\mathcal{D}_{N}$ of $A \times G$ and $\operatorname{im} j_{A} \times j_{G} \mid \subset$ $M(A \times G)$, respectively, such that $\mathcal{D}$ is a (full) pre-Hilbert $\mathcal{D}_{N}$-module under right multiplication and inner product

$$
\langle x, y\rangle_{\mathcal{D}_{N}}=\int_{N} \hat{\delta}_{n}\left(x^{*} y\right) d n \quad \text { for } x, y \in \mathcal{D},
$$

the integral converging strictly in $M(A \times G)$. Let $Y_{G / N}^{G}$ denote the completion of the pre-Hilbert module $\mathcal{D}$. Then $Y_{G / N}^{G}$ has a left $A \times G \times{ }_{r} N$-module action determined by left multiplication on $\mathcal{D}$ and

$$
n \cdot x=\Delta(n)^{\frac{1}{2}} \hat{\delta}_{n}(x) \quad \text { for } n \in N, x \in \mathcal{D} .
$$

When $N$ is amenable and $\delta$ is a reduced coaction, Mansfield [Man91, Theorem 27] proves that $A \times G \times N$ is Morita equivalent to $A \times G / N$. For nonamenable subgroups and full coactions, the corresponding result is:

Theorem 2.1 ([KQ, Corollary 3.4]). Let $(A, G, \delta)$ be a nondegenerate coaction and $N$ a closed normal subgroup of $G$ such that

$$
j_{A} \times j_{G} \mid: A \times G / N \rightarrow M(A \times G)
$$

is faithful. Then $Y_{G / N}^{G}$ is an $A \times G \times{ }_{r} N-A \times G / N$ imprimitivity bimodule.

In view of the above theorem, if $\delta$ is a nondegenerate coaction and $N$ is a closed normal subgroup of $G$, we say Mansfield imprimitivity works for $N$ and $\delta$ whenever $j_{A} \times j_{G} \mid: A \times G / N \rightarrow M(A \times G)$ is faithful [KQ, Definition 3.5]. When Mansfield imprimitivity works we let $\langle\cdot, \cdot\rangle_{A \times G / N}$ denote the extension to $Y_{G / N}^{G}$ of the inner product $\langle\cdot, \cdot\rangle_{\mathcal{D}_{N}}$ on $\mathcal{D}$. Mansfield's computations show that the left inner product $A \times G \times{ }_{r} N\langle x, y\rangle$ for $x, y \in \mathcal{D}$ can be identified with the element

$$
A \times G \times{ }_{r} N\langle x, y\rangle(n)=x \hat{\delta}_{n}\left(y^{*}\right) \Delta(n)^{-\frac{1}{2}}
$$

of $C_{c}(N, \mathcal{D})$. When $\delta$ is nondegenerate, Mansfield imprimitivity works if either $N$ is amenable or $\delta$ is normal [KQ, Lemma 3.2]. If Mansfield imprimitivity works for $N$ and $\delta$, then it also works for any closed subgroup contained in $N$ [KQ, 
Theorem 5.2] and any coaction Morita equivalent to $\delta$ [KQ, Theorem 5.3]. If $K$ is a closed normal subgroup of $G$ containing $N$ and $(A, K, \epsilon)$ is a coaction, then Mansfield imprimitivity works for $N$ and $\epsilon$ if and only if it works for $N$ and the inflated coaction $(A, G, \operatorname{Inf} \epsilon)[\mathrm{KQ}$, Theorem 5.4].

When we say $(A, G, G / K, \delta, \tau)$ is a twisted coaction, we mean $K$ is a closed normal subgroup of $G$ and $\tau: C_{0}(G / K) \rightarrow M(A)$ is a twist for $\delta$ over $G / K$ [PR94]. We let $I_{\tau}$ denote the twisting ideal of $A \times G, A \times_{G / K} G=(A \times G) / I_{\tau}$ the twisted crossed product, and $\tilde{\delta}$ the dual action of $K$ on $A \times{ }_{G / K} G$ (see Lemma 4.4 below). If further $N$ is a closed normal subgroup of $G$ contained in $K$, then $\tau$ is also a twist for the restricted coaction $(A, G / N, \delta \mid)$ over the quotient $G / K \cong(G / N) /(K / N)$. Let $I_{\tau}^{N}$ denote the twisting ideal of $A \times G / N$. Then there is a restricted twisted crossed product $A \times_{G / K} G / N=(A \times G / N) / I_{\tau}^{N}$. The following result generalizes [PR94, Theorem 4.1]:

Theorem 2.2. [KQ, Theorem 4.4] Let $(A, G, G / K, \delta, \tau)$ be a nondegenerate twisted coaction and $N$ a closed normal subgroup of $G$ contained in $K$ such that Mansfield imprimitivity works for $N$ and $\delta$. Then the quotient $Z_{G / N}^{G}=Y_{G / N}^{G} /\left(Y_{G / N}^{G} \cdot I_{\tau}^{N}\right)$ is an $A \times_{G / K} G \times_{r} N-A \times_{G / K} G / N$ imprimitivity bimodule.

If $(A, G, G / K, \delta, \tau)$ is a nondegenerate twisted coaction, then Mansfield imprimitivity works for $K$ and $\delta$ if and only if $\delta$ is normal [KQ, Lemma 3.6]. If $(A, G, G / K, \delta, \tau)$ is a nondegenerate normal twisted coaction and $N$ is a closed normal subgroup of $G$ contained in $K$, then Mansfield imprimitivity works for $N$ and $\delta$ since $\delta$ is normal, and also for $N$ and the Morita equivalent stabilized coaction $\left(A \times_{G / K} G \times{ }_{r} K, K,(\widehat{\widetilde{\delta}})^{\mathrm{n}}\right)$ [ER96, Theorem 3.1], [KQ, Theorem 5.5].

Hilbert Modules and Rieffel Induction. Everything in this paper revolves around Rieffel's induction process, so we should make our conventions explicit. For more detailed treatments of this material we refer the reader to [Lan95], [Rie74], [KQ]. All our Hilbert modules will be full, i.e., the closed span of the inner product generates the $C^{*}$-algebra. If $X$ is a right Hilbert $B$-module and $A$ acts nondegenerately on $X$ by adjointable $B$-module maps (so there is a homomorphism $A \rightarrow \mathcal{L}_{B}(X)$ such that $\left.A X=X\right)$, we say $X$ is a right-Hilbert $A-B$ bimodule. (This terminology first appears in [Bui95].) If $X$ is also a left Hilbert $A$-module such that ${ }_{A}\langle x, y\rangle \cdot z=x \cdot\langle y, z\rangle_{B}$ for $x, y, z \in X$, then of course $X$ is an $A-B$ imprimitivity bimodule. We denote the reverse bimodule by $\tilde{X}$, with elements $\tilde{x}$.

When $X$ is a right-Hilbert $A-B$ bimodule, Rieffel induction gives a functor

$$
X-\operatorname{Ind}_{B}^{A}: \operatorname{Rep} B \rightarrow \operatorname{Rep} A,
$$

and we leave out parts of the notation if confusion seems unlikely. Actually, $X-\operatorname{Ind}_{B}^{A}$ can be factored as

$$
X \text {-Ind: } \operatorname{Rep} B \rightarrow \operatorname{Rep} \mathcal{K}_{B}(X)
$$

followed by "restriction" from $\operatorname{Rep} \mathcal{K}_{B}(X)$ to $\operatorname{Rep} A$. Since we will need it a lot, we abstract this latter bit: if $\pi: A \rightarrow M(B)$ is a nondegenerate homomorphism, composition with $\pi$ gives a "restriction" map

$$
\operatorname{Res}_{B}^{A}=\pi^{*}: \operatorname{Rep} B \rightarrow \operatorname{Rep} A .
$$


We view this as a Rieffel induction process: $B$ becomes a right-Hilbert $A-B$ bimodule via

$$
\begin{aligned}
\langle b, c\rangle_{B} & =b^{*} c, \\
b \cdot c & =b c \\
a \cdot b & =\pi(a) b,
\end{aligned}
$$

for $a \in A, b, c \in B$.

Now suppose $\pi: A \rightarrow M(B)$ is a nondegenerate homomorphism and $X$ is a right-Hilbert $B-C$ bimodule. Then $X$ becomes a right-Hilbert $A-C$ bimodule via

$$
a \cdot x=\pi(a) x \quad \text { for } a \in A, x \in X .
$$

On the other hand, we can regard $B$ as a right-Hilbert $A-B$ bimodule, and the map $b \otimes x \mapsto b x$ induces an isomorphism

$$
B \otimes_{B} X \rightarrow X
$$

of right-Hilbert $A-C$ bimodules. In particular, we get a commutative diagram

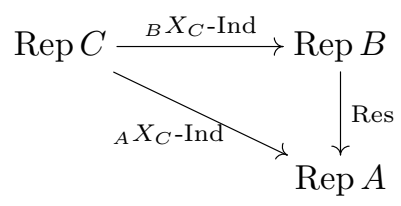

where we use prescripts and postscripts to indicate the coefficient algebras when necessary. In general, we will omit parts of the notation, so that when we say

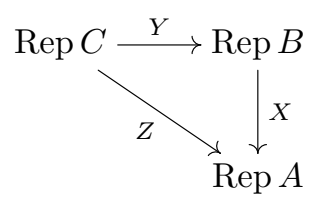

is a commutative diagram, we mean $X$ is a right-Hilbert $A-B$ bimodule, and similarly for $Y$ and $Z$, and the equation $(X$-Ind $) \circ(Y$-Ind $)=Z$-Ind holds in the strong sense that

$$
X \otimes_{B} Y \cong Z
$$

as right-Hilbert $A-C$ bimodules.

Recall that Rieffel induction gives rise to maps between ideals, so if $I$ is an ideal of $B$ and if $\pi$ is any nondegenerate representation of $B$ with kernel $I$, then $X$-Ind $I$ is the kernel of $X$-Ind $\pi$. When we have a commutative diagram of Hilbert modules as in (2.2), we of course get

$$
(X \text {-Ind }) \circ(Y \text {-Ind })=Z \text {-Ind }
$$

as maps from ideals of $C$ to ideals of $A$.

We will often want to pass from a commutative diagram of Hilbert modules to quotients. There is a subtle point that needs checking:

Lemma 2.3. Let $X$ and $Y$ be right-Hilbert $A-B$ and right-Hilbert $B-C$ bimodules, respectively, and let $K$ be an ideal of $C$. Further, let $J=Y$-Ind $K$ and $I=X$-Ind $J$ be the corresponding induced ideals of $B$ and $A$, respectively, and let $q_{X}: X \rightarrow X /(X \cdot J)$ and $q_{Y}: Y \rightarrow Y /(Y \cdot K)$ be the quotient maps. Then 
$q_{X} \otimes q_{Y}$ induces an isomorphism between the right-Hilbert $A / I-C / K$ bimodules $\left(X \otimes_{B} Y\right) /\left(\left(X \otimes_{B} Y\right) \cdot K\right)$ and $(X /(X \cdot J)) \otimes_{B / J}(Y /(Y \cdot K))$.

Proof. Straightforward; a slightly different version was given in [Rae81, Lemma 1.10].

Corollary 2.4. Suppose the diagram

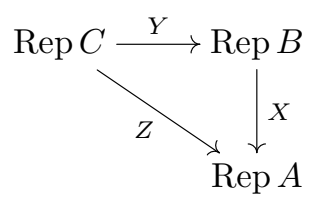

commutes in the usual strong sense that $Z \cong X \otimes_{B} Y$. Further suppose that $K$ is an ideal of $C$, and set $J=Y$-Ind $K$ and $I=X$-Ind $J$. Then the diagram

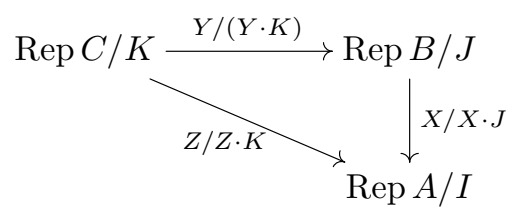

also commutes in the usual strong sense.

Proof. Since $Z \cong X \otimes_{B} Y$, certainly

$$
Z /(Z \cdot K) \cong\left(X \otimes_{B} Y\right) /\left(\left(X \otimes_{B} Y\right) \cdot K\right),
$$

so the above lemma immediately gives the corollary.

Similarly, commutative diagrams with any number of vertices pass to quotients.

Green Induction for Reduced Crossed Products. We will need an induction process for reduced crossed products by actions. We could deduce it from Green's version by applying [QS92], but we give a direct argument since we need the explicit imprimitivity bimodule.

Let $(B, G, \alpha)$ be an action and $H$ a closed subgroup of $G$. Recall that Green's inducing process starts with the right-pre-Hilbert $C_{c}(G, B)-C_{c}(H, B)$ bimodule $C_{c}(G, B)$, where the operations for $f, x, y \in C_{c}(G, B), g \in C_{c}(H, B)$ are given by

$$
\begin{aligned}
(f \cdot x)(t) & =\int_{G} f(s) \alpha_{s}\left(x\left(s^{-1} t\right)\right) \Delta_{G}(s)^{\frac{1}{2}} d s, \\
(x \cdot g)(t) & =\int_{H} x\left(t h^{-1}\right) \alpha_{t h^{-1}}(g(h)) \Delta_{H}(h)^{-\frac{1}{2}} d h, \\
\langle x, y\rangle_{C_{c}(H, B)}(h) & =\int_{G} \alpha_{s}\left(x\left(s^{-1}\right)^{*} y\left(s^{-1} h\right)\right) \Delta_{H}(h)^{-\frac{1}{2}} d s .
\end{aligned}
$$

The particular arrangement of modular functions comes from [Rae88]; Green uses unorthodox conventions. Let $Z_{H}^{G}$ be the completion of the pre-Hilbert module $C_{c}(G, B)$, so $Z_{H}^{G}$ is a right-Hilbert $B \times G-B \times H$ bimodule. We need to know that the kernels of the regular representations match up, so that $Z_{H}^{G}$ passes to a right-Hilbert $B \times{ }_{r} G-B \times{ }_{r} H$ bimodule. 
Lemma 2.5. Let $(B, G, \alpha)$ be an action, $H$ a closed subgroup of $G, Z_{H}^{G}$ Green's right-Hilbert $B \times G-B \times H$ bimodule, and $I$ the kernel of the regular representation of $B \times H$. Then the induced ideal $Z_{H}^{G}$-Ind $\operatorname{In}_{B \times H}^{B \times G} I$ is the kernel of the regular representation of $B \times G$. Consequently, the quotient $X_{H}^{G}=Z_{H}^{G} /\left(Z_{H}^{G} \cdot I\right)$ is a right-Hilbert $B \times{ }_{r} G-B \times{ }_{r} H$ bimodule.

Proof. This follows from induction in stages: the proof of [Gre78, Proposition 8] shows that the diagram

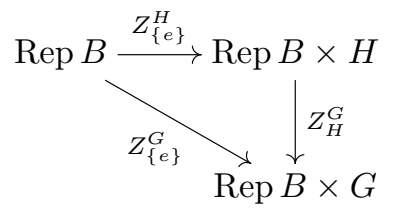

commutes in the usual strong sense. If $\pi$ is any faithful representation of $B$, then $Z_{\{e\}}^{H}$-Ind $\pi$ and $Z_{\{e\}}^{G}$-Ind $\pi$ are the regular representations of $B \times H$ and $B \times G$, respectively. So the ideals $Z_{\{e\}}^{H}-\operatorname{Ind}\{0\}$ and $Z_{\{e\}}^{G}-\operatorname{Ind}\{0\}$ are the kernels of the regular representations. By commutativity of diagram (2.5),

$$
Z_{\{e\}}^{G}-\operatorname{Ind}\{0\}=Z_{H^{G}}^{G} \operatorname{Ind}\left(Z_{\{e\}}^{H}-\operatorname{Ind}\{0\}\right),
$$

so indeed $Z_{H}^{G}$ induces the kernel $I$ of $B \times H \rightarrow B \times{ }_{r} H$ to the kernel of $B \times G \rightarrow$ $B \times{ }_{r} G$.

Corollary 2.6. Let $(B, G, \alpha)$ be an action, $H$ a closed subgroup of $G$, and $X_{H}^{G}$ the right-Hilbert $B \times{ }_{r} G-B \times{ }_{r} H$ bimodule obtained in Lemma 2.5. Then the diagram

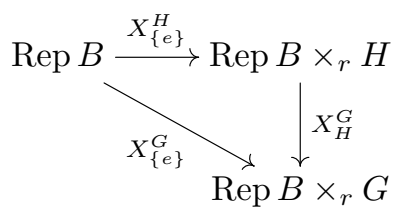

commutes in the usual strong sense.

Proof. This follows from the above lemma and Corollary 2.4.

We emphasize that $X_{H}^{G}$ may be viewed as the completion of $C_{c}(G, B)$ with respect to the norm induced by the $B \times_{r} H$-valued pre-inner product. In particular, the actions of $B \times{ }_{r} H$ and $B \times{ }_{r} G$ on $X_{H}^{G}$ are determined by the covariant representations of $(B, H, \alpha)$ and $(B, G, \alpha)$ on $C_{c}(G, B)$.

\section{Mansfield Restriction and Green induCtion}

Suppose we have a twisted coaction $(A, G, G / K, \delta, \tau)$, and closed normal subgroups $N \subset H$ of $G$ contained in $K$. In this section, we show that when Mansfield imprimitivity works, the following diagram commutes in the usual strong sense:

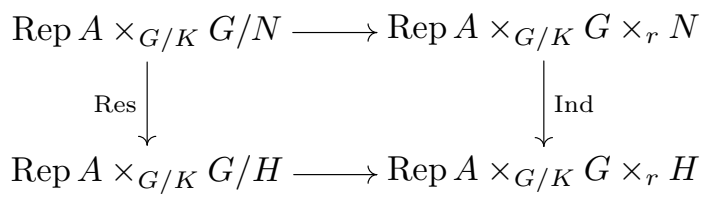


We will do this in two steps, first showing that the analogous untwisted diagram commutes, and then showing that the twisting ideals in the various crossed products match up properly, so that commutativity is preserved on taking quotients by these ideals.

For $N=\{e\}$, a weak form of the following theorem was proven in $[\mathrm{KQ}$, Theorem 4.1].

Theorem 3.1. Let $(A, G, \delta)$ be a nondegenerate coaction, and let $N \subset H$ be closed normal subgroups of $G$ such that Mansfield imprimitivity works for $H$ (which is automatic if $H$ is amenable). Then the diagram

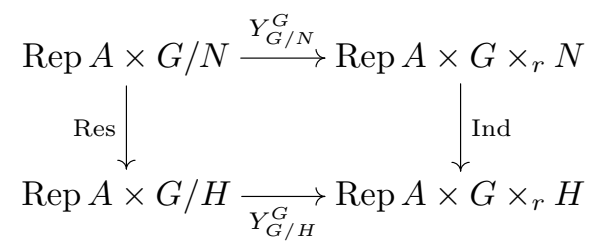

commutes in the usual strong sense.

Proof. First note that by [KQ, Theorem 5.2], Mansfield imprimitivity also works for $N$ and $\delta$, so $Y_{G / N}^{G}$ is indeed an $A \times G \times{ }_{r} N-A \times G / N$ imprimitivity bimodule, and the above diagram makes sense.

We shall actually prove that the Hilbert module tensor product

$$
Y_{G / H}^{G} \otimes_{A \times G / H} \widetilde{Y_{G / N}^{G}}
$$

of Mansfield bimodules is isomorphic to the reduction $X_{N}^{H}$ of Green's bimodule, as a right-Hilbert $A \times G \times_{r} H-A \times G \times_{r} N$ bimodule. This suffices, because then

$$
\begin{aligned}
X_{N}^{H} \otimes_{A \times G \times{ }_{r} N} Y_{G / N}^{G} & \cong Y_{G / H}^{G} \otimes_{A \times G / H} \widetilde{Y_{G / N}^{G}} \otimes_{A \times G \times{ }_{r} N} Y_{G / N}^{G} \\
& \cong Y_{G / H}^{G} \otimes_{A \times G / H}(A \times G / N)
\end{aligned}
$$

as a right-Hilbert $A \times G \times{ }_{r} H-A \times G / N$ bimodule, and this is exactly what it means for the above diagram to commute in the strong sense.

Both bimodules in the tensor product (3.2) are completions of Mansfield's dense subalgebra $\mathcal{D}$ of $A \times G$ for the appropriate inner products. Our isomorphism will be the extension to $Y_{G / H}^{G} \otimes_{A \times G / H} \widetilde{Y_{G / N}^{G}}$ of the map $\Phi: \mathcal{D} \otimes \tilde{\mathcal{D}} \rightarrow C_{c}(H, \mathcal{D})$ defined by

$$
\Phi(x \otimes \tilde{y})(h)=x \hat{\delta}_{h}\left(y^{*}\right) .
$$

Note that, up to a modular function, $\Phi(x \otimes \tilde{y})$ is just Mansfield's left $C_{c}(H, \mathcal{D})$ valued inner product $C_{c}(H, \mathcal{D})\langle x, y\rangle$, and hence does indeed give an element of $C_{c}(H, \mathcal{D})$. In fact, if we define $f^{\prime}(h)=f(h) \Delta_{H}(h)^{-\frac{1}{2}}$, then the map $f \mapsto f^{\prime}$ is a homeomorphism of $C_{c}(H, \mathcal{D})$ (with the inductive limit topology) onto itself, which takes $\Phi(\mathcal{D} \odot \mathcal{D})$ to $C_{c}(H, \mathcal{D})\langle\mathcal{D}, \mathcal{D}\rangle$. This latter set is dense in $C_{c}(H, A \times G)$ for the inductive limit topology ([Man91, Lemma 25]); it follows that the range of $\Phi$ is also inductive limit dense in $C_{c}(H, A \times G)$, and therefore in $X_{N}^{H}$.

It only remains to show that $\Phi$ preserves the Hilbert module structure. For the left action of $A \times G \times{ }_{r} H$, fix $d \in \mathcal{D} \subset A \times G$ and $h, t \in H$. Then:

$$
d \cdot \Phi(x \otimes \tilde{y})(h)=d x \hat{\delta}_{h}\left(y^{*}\right)=\Phi(d \cdot x \otimes \tilde{y})
$$


and

$$
\begin{aligned}
t \cdot \Phi(x \otimes \tilde{y})(h) & =\hat{\delta}_{t}\left(\Phi(x \otimes \tilde{y})\left(t^{-1} h\right)\right) \Delta(t)^{\frac{1}{2}} \\
& =\hat{\delta}_{t}\left(x \hat{\delta}_{t^{-1} h}\left(y^{*}\right)\right) \Delta(t)^{\frac{1}{2}} \\
& =\hat{\delta}_{t}(x) \hat{\delta}_{h}\left(y^{*}\right) \Delta(t)^{\frac{1}{2}} \\
& =(t \cdot x) \hat{\delta}_{h}\left(y^{*}\right) \\
& =\Phi(t \cdot x \otimes \tilde{y})(h) .
\end{aligned}
$$

For the right action of $A \times G \times{ }_{r} N$, fix $d \in \mathcal{D} \subset A \times G, h \in H$ and $n \in N$; then one similarly verifies that

$$
\begin{aligned}
\Phi(x \otimes \tilde{y}) \cdot d(h) & =x \hat{\delta}_{h}\left(\left(d^{*} y\right)^{*}\right) \\
& =\Phi(x \otimes \tilde{y} \cdot d)(h)
\end{aligned}
$$

and

$$
\begin{aligned}
\Phi(x \otimes \tilde{y}) \cdot n(h) & =x \hat{\delta}_{h}\left(\hat{\delta}_{n^{-1}}\left(y^{*}\right) \Delta(n)^{-\frac{1}{2}}\right) \\
& =\Phi(x \otimes \tilde{y} \cdot n)(h) .
\end{aligned}
$$

For the right $A \times G \times{ }_{r} N$-valued inner product, fix $n \in N$ and compute:

$$
\begin{aligned}
\langle\Phi(x & \otimes \tilde{y}), \Phi(z \otimes \tilde{w})\rangle_{A \times G \times_{r} N}(n) \\
& =\int_{H} \hat{\delta}_{h}\left(\Phi(x \otimes \tilde{y})\left(h^{-1}\right)^{*} \Phi(z \otimes \tilde{w})\left(h^{-1} n\right)\right) \Delta(n)^{-\frac{1}{2}} d h \\
& =\int_{H} \hat{\delta}_{h}\left(\left(x \hat{\delta}_{h^{-1}}\left(y^{*}\right)\right)^{*} z \hat{\delta}_{h^{-1} n}\left(w^{*}\right)\right) \Delta(n)^{-\frac{1}{2}} d h \\
& =\int_{H} y \hat{\delta}_{h}\left(x^{*} z\right) \hat{\delta}_{n}\left(w^{*}\right) d h \Delta(n)^{-\frac{1}{2}} \\
h \mapsto n h & \int_{H} y \hat{\delta}_{n}\left(\hat{\delta}_{h}\left(x^{*} z\right) w^{*}\right) d h \Delta(n)^{-\frac{1}{2}} \\
& =y \hat{\delta}_{n}\left(w \int_{H} \hat{\delta}_{h}\left(z^{*} x\right) d h\right)^{*} \Delta(n)^{-\frac{1}{2}} \\
& =y \hat{\delta}_{n}\left(\left(w \cdot\langle z, x\rangle_{A \times G / H}\right)^{*}\right) \Delta(n)^{-\frac{1}{2}} \\
& =\quad A \times G \times{ }_{r} N\left\langle y, w \cdot\langle z, x\rangle_{A \times G / H}\right\rangle(n) \\
& =\quad\langle x \otimes \tilde{y}, z \otimes \tilde{w}\rangle_{A \times G \times{ }_{r} N}(n) .
\end{aligned}
$$

It now follows that $\Phi$ is a right-Hilbert $A \times G \times{ }_{r} H-A \times G \times{ }_{r} N$ bimodule isomorphism of $Y_{G / H}^{G} \otimes_{A \times G / H} \widetilde{Y_{G / N}^{G}}$ onto $X_{N}^{H}$.

Corollary 3.2. Let $(A, G, G / K, \delta, \tau)$ be a nondegenerate twisted coaction, and let $N \subset H$ be closed normal subgroups of $G$ contained in $K$ such that Mansfield imprimitivity works for $H$ and $\delta$ (which is automatic if $H$ is amenable). Then the 
diagram

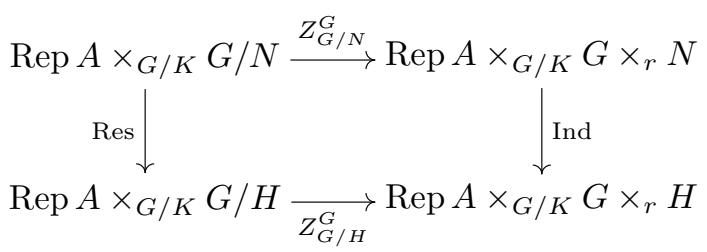

commutes in the usual strong sense.

Proof. We show the appropriate ideals in diagram (3.1) match up and appeal to Corollary 2.4; because the diagram commutes, and the top and bottom maps are Morita equivalences, we need only match up the ideals along three sides. Let $I_{\tau}$, $I_{\tau}^{N}$, and $I_{\tau}^{H}$ be the twisting ideals of $A \times G, A \times G / N$, and $A \times G / H$, respectively. The reduced crossed product $A \times{ }_{G / K} G \times{ }_{r} N$ is by definition $\left((A \times G) / I_{\tau}\right) \times_{r} N$. If $\pi$ is a representation of $A \times G$ with kernel $I_{\tau}$ (for example, if $\pi=k_{A} \times k_{G}$ ), then $\pi$ induces a faithful representation $X_{\{e\}}^{N}$-Ind $\pi$ of $\left((A \times G) / I_{\tau}\right) \times_{r} N$; thus

$$
A \times{ }_{G / K} G \times{ }_{r} N=\left(A \times G \times{ }_{r} N\right) /\left(X_{\{e\}}^{N} \text {-Ind } I_{\tau}\right) .
$$

It is part of the content of $[\mathrm{KQ}$, Theorem 4.4] that

$$
Y_{G / N^{-}}^{G} \text { Ind } I_{\tau}^{N}=X_{\{e\}}^{N} \text {-Ind } I_{\tau}
$$

(see [KQ, Equation 4.2]). Hence the appropriate ideals match up along the top of diagram (3.1), and similarly along the bottom.

Since

$$
X_{N}^{H}-\operatorname{Ind}\left(X_{\{e\}}^{N} \text {-Ind } I_{\tau}\right)=X_{\{e\}}^{H} \text {-Ind } I_{\tau},
$$

by Corollary 2.6, the ideals also match up along the right side of diagram (3.1).

\section{Mansfield induction and Green Restriction}

In this section we prove analogs of Theorem 3.1 and Corollary 3.2, where now we use Mansfield induction on the left sides of the diagrams and restriction on the right. As in the previous section, we first prove an untwisted version, and then show that the twisting ideals match up properly.

Theorem 4.1. Let $(A, G, \delta)$ be a nondegenerate coaction, and let $N \subset H$ be closed normal subgroups of $G$ such that Mansfield imprimitivity works for $H$ (which is automatic if $H$ is amenable). Then the diagram

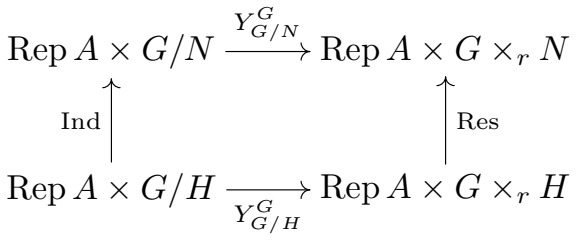

commutes in the usual strong sense.

Proof. Let us denote the Hilbert module for Mansfield induction from $A \times G / H$ to $A \times G / N$ by $Y_{G / H}^{G / N}$; here we are identifying $G / H$ with $(G / N) /(H / N)$, so $Y_{G / H}^{G / N}$ is 
a completion of Mansfield's dense subalgebra $\mathcal{D}_{N}$ of $A \times G / N$. We shall prove that the Hilbert module tensor product

$$
Y_{G / N}^{G} \otimes_{A \times G / N} Y_{G / H}^{G / N}
$$

is isomorphic to $Y_{G / H}^{G}$ as a right-Hilbert $A \times G \times{ }_{r} N-A \times G / H$ bimodule. This suffices, because then

$$
Y_{G / N}^{G} \otimes_{A \times G / N} Y_{G / H}^{G / N} \cong\left(A \times G \times{ }_{r} H\right) \otimes_{A \times G \times{ }_{r} H} Y_{G / H}^{G}
$$

as a right-Hilbert $A \times G \times{ }_{r} N-A \times G / H$ bimodule, and this is exactly what it means for the above diagram to commute in the strong sense.

Our map will be the extension to $Y_{G / N}^{G} \otimes_{A \times G / N} Y_{G / H}^{G / N}$ of the map $\Psi: \mathcal{D} \otimes \mathcal{D}_{N} \rightarrow \mathcal{D}$ defined by

$$
\Psi(x \otimes y)=x y
$$

here the product $x y$ makes sense in $M(A \times G)$ because $y$ belongs to $\mathcal{D}_{N} \subset M(A \times G)$. In other words, this product is given by the right action of $\mathcal{D}_{N}$ on Mansfield's bimodule $\mathcal{D}$, and hence $\Psi(x \otimes y)$ does indeed belong to $\mathcal{D}$.

We now show that $\Psi$ preserves the right-Hilbert bimodule structure. Since $A \times G$ on the left and $A \times G / H$ on the right act by multiplication in $M(A \times G)$, it is immediate that $\Psi$ preserves these actions. To see that $\Psi$ preserves the left $N$ action is a straightforward calculation, using the fact that each $y \in \mathcal{D}_{N}$ is fixed by $\hat{\delta}_{n}$ for $n \in N$ :

$$
\Psi(n \cdot x \otimes y)=\hat{\delta}_{n}(x) y \Delta(n)^{\frac{1}{2}}=n \cdot \Psi(x \otimes y) .
$$

To see that $\Psi$ preserves the right $A \times G / H$-valued inner products, note that $\left.\widehat{\delta}\right|_{t N}=$ $\hat{\delta}_{t}$ on $A \times G / N$, and compute:

$$
\begin{aligned}
\langle\Psi & (x \otimes y), \Psi(z \otimes w)\rangle_{A \times G / H} \\
& =\int_{H} \hat{\delta}_{t}\left(\Psi(x \otimes y)^{*} \Psi(z \otimes w)\right) d t \\
& =\int_{H} \hat{\delta}_{t}\left((x y)^{*} z w\right) d t \\
& =\int_{H / N} \int_{N} \hat{\delta}_{t n}\left(y^{*} x^{*} z w\right) d n d t N \\
& =\int_{H / N} \int_{N} \hat{\delta}_{t}\left(y^{*} \hat{\delta}_{n}\left(x^{*} z\right) w\right) d n d t N \\
& =\int_{H / N} \hat{\delta}_{t}\left(y^{*}\langle x, z\rangle_{A \times G / N} w\right) d t N \\
& =\int_{H / N} \hat{\delta}_{t N}\left(y^{*}\langle x, z\rangle_{A \times G / N} \cdot w\right) d t N \\
& =\left\langle y,\langle x, z\rangle_{A \times G / N} \cdot w\right\rangle_{A \times G / H} \\
& =\langle x \otimes y, z \otimes w\rangle_{A \times G / H} .
\end{aligned}
$$

It only remains to show that the range of $\Psi$ is dense in $Y_{G / H}^{G}$. For this, note that $\mathcal{D} \cdot j_{G}\left(C_{c}(G / N)\right)=\mathcal{D}$, since if $x \in \mathcal{D}$ is $(u, E)$, we may choose $f \in C_{c}(G / N)$ such that $f$ is identically 1 on $E$, and then $x \cdot j_{G}(f)=x$. Hence, $\mathcal{D} \cdot j_{G}\left(C_{c}(G / N)\right)$ 
is dense in $Y_{G / H}^{G}$. Since $Y_{G / H}^{G}$ is a nondegenerate right $A$-module, and $\delta_{A_{c}(G)}(A)$ is dense in $A$, we therefore have

$$
\begin{aligned}
\overline{\Phi\left(\mathcal{D} \odot \mathcal{D}_{N}\right)} & =\overline{\mathcal{D} \cdot \mathcal{D}_{N}} \\
& =\overline{\mathcal{D} \cdot j_{G}\left(C_{c}(G / N)\right) j_{A}\left(\delta_{A_{c}(G)}(A)\right)} \\
& =\overline{\mathcal{D} \cdot j_{A}\left(\delta_{A_{C}(G)}(A)\right)} \\
& =Y_{G / H}^{G} .
\end{aligned}
$$

It now follows that $\Psi$ is a right-Hilbert $A \times G \times{ }_{r} N-A \times G / H$ bimodule isomorphism of $Y_{G / N}^{G} \otimes_{A \times G / N} Y_{G / H}^{G / N}$ onto $Y_{G / H}^{G}$.

Corollary 4.2 (Mansfield Induction in Stages). Let $(A, G, \delta)$ be a nondegenerate coaction, and let $N \subset H$ be closed normal subgroups of $G$ such that Mansfield imprimitivity works for $H$ (which is automatic if $H$ is amenable). Then the diagram

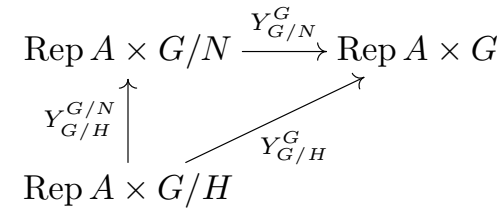

commutes in the usual strong sense.

Proof. The corollary requires that the Hilbert module tensor product

$$
Y_{G / N}^{G} \otimes_{A \times G / N} Y_{G / H}^{G / N}
$$

be isomorphic to $Y_{G / H}^{G}$ as a right-Hilbert $A \times G-A \times G / H$ bimodule; we showed slightly more than this in the proof of Theorem 4.1 by including the left $N$-action.

Corollary 4.3. Let $(A, G, G / K, \delta, \tau)$ be a nondegenerate twisted coaction, and let $N \subset H$ be closed normal subgroups of $G$ contained in $K$ such that Mansfield imprimitivity works for $H$ and $\delta$ (which is automatic if $H$ is amenable). Then the diagram

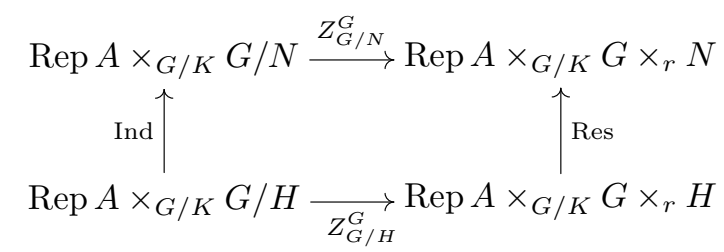

commutes in the usual strong sense.

Proof. As with Corollary 3.2, we need only show the appropriate ideals in the diagram (4.1) match up, and appeal to Corollary 2.4. Moreover, the top and bottom of diagram (4.1) are the same as in diagram (3.1); the ideals were shown to match up along these arrows in the proof of Corollary 3.2. Hence it only remains to see that the ideals match up along the left side of diagram (4.1).

As before, let $I_{\tau}, I_{\tau}^{N}$, and $I_{\tau}^{H}$ be the twisting ideals of $A \times G, A \times G / N$, and $A \times G / H$, respectively. We need

$$
\operatorname{Ind}_{G / H}^{G / N} I_{\tau}^{H}=I_{\tau}^{N}
$$


Again recall from [KQ, Equation (4.2)] that

$$
Y_{G / H^{-}}^{G} \text { Ind } I_{\tau}^{H}=X_{\{e\}}^{H} \text {-Ind } I_{\tau} .
$$

Restricting from $A \times G \times{ }_{r} H$ to $A \times G$, we have

$$
\operatorname{Res}_{A \times G \times{ }_{r} H}^{A \times G} Y_{G / H^{-}}^{G} \text { Ind } I_{\tau}^{H}=\operatorname{Res}_{A \times G \times{ }_{r} H}^{A \times G} X_{\{e\}}^{H} \text {-Ind } I_{\tau},
$$

and the left-hand ideal is by definition $\operatorname{Ind}_{G / H}^{G} I_{\tau}^{H}$. We claim that the right-hand ideal is just $I_{\tau}$. By (the reduced version of) [Gre78, Proposition 11] it suffices to show that $I_{\tau}$ is invariant under the action $\left.\hat{\delta}\right|_{H}$ of $H$; as the following lemma shows, $I_{\tau}$ is in fact invariant under $\left.\hat{\delta}\right|_{K}$.

Lemma 4.4. Let $(A, G, G / K, \delta, \tau)$ be a twisted coaction. Then the twisting ideal $I_{\tau} \subset A \times G$ is invariant for the dual action $\left.\hat{\delta}\right|_{K}$ of $K$.

Proof. Since

$$
I_{\tau}=\bigcap\{\operatorname{ker} \pi \times \mu \mid(\pi, \mu) \text { preserves } \tau\}
$$

and

$$
(\pi \times \mu) \circ \hat{\delta}_{k}=\pi \times\left(\mu \circ \sigma_{k}\right)
$$

where $\sigma_{k}(f)(s)=f(s k)$, it suffices to show that $\left(\pi, \mu \circ \sigma_{k}\right)$ preserves $\tau$ whenever $(\pi, \mu)$ preserves $\tau$, for each $k \in K$. By [PR94, Remark 2.6], $(\pi, \mu)$ preserves $\tau$ if and only if $\pi \circ \tau=\left.\mu\right|_{C_{0}(G / K)}$; since $\left.\mu \circ \sigma_{k}\right|_{C_{0}(G / K)}=\left.\mu\right|_{C_{0}(G / K)}$ for each $k \in K$, we are done.

Thus far we have shown that

$$
\operatorname{Ind}_{G / H}^{G} I_{\tau}^{H}=I_{\tau}
$$

for a twisted coaction $(A, G, G / K, \delta, \tau)$ and a closed normal subgroup $H$ of $G$ contained in $K$. Viewing $\tau: C_{0}(G / K) \rightarrow M(A)$ as a map on $C_{0}((G / N) /(K / N))$, we apply this to the twisted coaction

$$
(A, G / N,(G / N) /(K / N), \delta \mid, \tau)
$$

and the subgroup $H / N \subset G / N$ to get

$$
\operatorname{Ind}_{(G / N) /(H / N)}^{G / N} I_{\tau}^{H / N}=I_{\tau}^{N}
$$

as ideals in $A \times G / N$. Now $A \times(G / N) /(H / N)=A \times G / H$, and the respective twists are really the same; hence the twisting ideals $I_{\tau}^{H / N}$ and $I_{\tau}^{H}$ are the same, so

$$
\operatorname{Ind}_{(G / N) /(H / N)}^{G / N} I_{\tau}^{H / N}=\operatorname{Ind}_{G / H}^{G / N} I_{\tau}^{H} .
$$

This establishes (4.2), which completes the proof of Corollary 4.3.

\section{Morita Equivalence, inflation, And Stabilization}

In this section we show that our Res-Ind duality is compatible with certain standard constructions.

Before discussing Morita equivalence of coactions, we recall the concept of multiplier bimodules introduced in [ER95]. A multiplier $m=\left(m_{A}, m_{B}\right)$ of ${ }_{A} X_{B}$ consists of an $A$-linear map $m_{A}: A \rightarrow X$ and a $B$-linear map $m_{B}: B \rightarrow X$ such that $m_{A}(a) b=a m_{B}(b)$ for $a \in A, b \in B$. The multiplier bimodule $M(X)$ consists of all multipliers of $X$. An imprimitivity bimodule homomorphism $\varphi=$ $\left(\varphi_{A}, \varphi_{X}, \varphi_{B}\right):{ }_{A} X_{B} \rightarrow M\left({ }_{C} Y_{D}\right)$ consists of homomorphisms $\varphi_{A}: A \rightarrow M(C)$ and 
$\varphi_{B}: B \rightarrow M(D)$ and a bimodule map $\varphi_{X}: X \rightarrow M(Y)$ preserving the inner products:

$$
\begin{gathered}
M(C)\left\langle\varphi_{X}(x), \varphi_{X}(y)\right\rangle=\varphi_{A}\left({ }_{A}\langle x, y\rangle\right) \quad \text { and } \\
\left\langle\varphi_{X}(x), \varphi_{X}(y)\right\rangle_{M(D)}=\varphi_{B}\left(\langle x, y\rangle_{B}\right) \quad \text { for } x, y \in X
\end{gathered}
$$

$\varphi$ is called nondegenerate if $\varphi_{A}$ and $\varphi_{B}$ are nondegenerate. Significantly, this implies an ostensibly stronger form of nondegeneracy:

Lemma 5.1. If $\varphi:{ }_{A} X_{B} \rightarrow M\left({ }_{C} Y_{D}\right)$ is a nondegenerate imprimitivity bimodule homomorphism, then

$$
\overline{C \varphi_{X}(X)}=Y=\overline{\varphi_{X}(X) D} .
$$

Proof. By symmetry, it suffices to show the second equality. Since $Y=\overline{C \cdot Y}$, and $\varphi_{A}$ is nondegenerate, we have $Y=\overline{\varphi_{A}(A) \cdot Y}$. Now, since the range of the inner product $A\langle\cdot, \cdot\rangle$ spans $A$, we have

$$
\begin{aligned}
Y & =\overline{\varphi_{A}\left({ }_{A}\langle X, X\rangle\right) \cdot Y} \\
& =\overline{M(C)\left\langle\varphi_{X}(X), \varphi_{X}(X)\right\rangle \cdot Y} \\
& =\overline{\varphi_{X}(X) \cdot\left\langle\varphi_{X}(X), Y\right\rangle_{M(D)}} .
\end{aligned}
$$

Because we can factor $Y=\overline{C \cdot Y}$, and $C \cdot M(Y) \subset Y$, the pairing $\langle\cdot, \cdot\rangle_{M(D)}$ takes $M(Y) \times Y$ to $D$, and we can deduce from our calculation that $Y \subset \overline{\varphi_{X}(X) \cdot D}$. The other inclusion is trivial, so this establishes the lemma.

Lemma 5.2. Let ${ }_{A} X_{B}$ and ${ }_{C} Y_{D}$ be imprimitivity bimodules, and let $\varphi: L(X) \rightarrow$ $M(L(Y))$ be a nondegenerate homomorphism such that

$$
\varphi\left(\begin{array}{ll}
1 & 0 \\
0 & 0
\end{array}\right)=\left(\begin{array}{ll}
1 & 0 \\
0 & 0
\end{array}\right)
$$

where $L(X)=\left(\begin{array}{cc}A & X \\ \tilde{X} & B\end{array}\right)$ is the linking algebra of $X$, and similarly for $Y$. Then $\varphi$ restricts on the corners to give a nondegenerate imprimitivity bimodule homomorphism $\Phi:{ }_{A} X_{B} \rightarrow M\left({ }_{C} Y_{D}\right)$.

Proof. Since $\varphi$ is nondegenerate, we have $\varphi\left(\begin{array}{ll}0 & 0 \\ 0 & 1\end{array}\right)=\left(\begin{array}{ll}0 & 0 \\ 0 & 1\end{array}\right)$. Since

$$
M(L(Y))=\left(\begin{array}{ll}
M(C) & M(Y) \\
M(\tilde{Y}) & M(D)
\end{array}\right),
$$

we deduce that there are unique linear maps

$$
\begin{gathered}
\Phi_{A}: A \rightarrow M(C), \quad \Phi_{X}: X \rightarrow M(Y), \\
\Phi_{\tilde{X}}: \tilde{X} \rightarrow M(\tilde{Y}), \quad \text { and } \quad \Phi_{B}: B \rightarrow M(D)
\end{gathered}
$$

such that

$$
\varphi=\left(\begin{array}{ll}
\Phi_{A} & \Phi_{X} \\
\Phi_{\tilde{X}} & \Phi_{B}
\end{array}\right)
$$

The algebraic properties of $\varphi$ show that $\Phi:=\left(\Phi_{A}, \Phi_{X}, \Phi_{B}\right)$ is an imprimitivity bimodule homomorphism of ${ }_{A} X_{B}$ to $M\left({ }_{C} Y_{D}\right)$, and nondegeneracy of $\Phi_{A}$ and $\Phi_{B}$ is inherited from nondegeneracy of $\varphi$.

We will need the following elementary fact about imprimitivity bimodule homomorphisms. 
Lemma 5.3. If $\varphi=\left(\varphi_{A}, \varphi_{X}, \varphi_{B}\right):{ }_{A} X_{B} \rightarrow M\left({ }_{C} Y_{D}\right)$ is a nondegenerate imprimitivity bimodule homomorphism, then the diagram

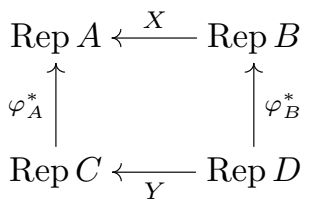

commutes in the usual strong sense.

Proof. We must show $X \otimes_{B} D \cong C \otimes_{C} Y$ as a right-Hilbert $A-D$ bimodule. Of course, ${ }_{A} C \otimes_{C} Y$ is isomorphic to ${ }_{A} Y$. Define $\Psi: X \odot D \rightarrow Y$ by

$$
\Psi(x \otimes d)=\varphi_{X}(x) d .
$$

The required properties of $\Psi$ follow from the nondegeneracy of $\varphi$, and straightforward calculations showing that

$$
\langle\Psi(x \otimes d), \Psi(y \otimes e)\rangle_{D}=\langle x \otimes d, y \otimes e\rangle_{D}
$$

and

$$
\Psi(a(x \otimes d) e)=a \Psi(x \otimes d) e
$$

for $a \in A, x, y \in X$, and $d, e \in D$.

Following [Ng95, Definition 3.3] (see also [BS89], [Bui94], [ER95]), a coaction $\delta$ of $G$ on an imprimitivity bimodule ${ }_{A} X_{B}$ is an imprimitivity bimodule homomorphism

$$
\delta=\left(\delta_{A}, \delta_{X}, \delta_{B}\right):{ }_{A} X_{B} \rightarrow M\left({ }_{A \otimes C^{*}(G)}\left(X \otimes C^{*}(G)\right)_{B \otimes C^{*}(G)}\right)
$$

such that $\left(A, G, \delta_{A}\right)$ and $\left(B, G, \delta_{B}\right)$ are $C^{*}$-coactions, and satisfying

$$
\left(\delta_{X} \otimes \mathrm{id}\right) \circ \delta_{X}=\left(\mathrm{id} \otimes \delta_{G}\right) \circ \delta_{X} .
$$

As a consequence of the definition, we automatically have $\delta_{X}(x) \cdot\left(1_{B} \otimes z\right)$ and $\left(1_{A} \otimes z\right) \cdot \delta_{X}(x) \in X \otimes C^{*}(G)$ for $x \in X, z \in C^{*}(G)$. Also, since by assumption $\delta_{A}$ and $\delta_{B}$ are nondegenerate $C^{*}$-homomorphisms, $\delta$ is automatically nondegenerate as an imprimitivity bimodule homomorphism. Hence, by Lemma 5.1, we have

$$
\overline{\delta_{X}(X) \cdot\left(B \otimes C^{*}(G)\right)}=\overline{\left(A \otimes C^{*}(G)\right) \cdot \delta_{X}(X)}=X \otimes C^{*}(G) .
$$

When such a $\delta$ exists we say $\left(A, G, \delta_{A}\right)$ and $\left(B, G, \delta_{B}\right)$ are Morita equivalent, and we call $\left(X, \delta_{X}\right)$ a Morita equivalence of $\delta_{A}$ and $\delta_{B}$.

If $N$ is a closed normal subgroup of $G$, then

$$
\delta_{X} \mid=\left(\operatorname{id} \otimes q_{N}\right) \circ \delta_{X}: X \rightarrow M\left(X \otimes C^{*}(G / N)\right)
$$

is a coaction of $G / N$ on $X$, where $q_{N}: C^{*}(G) \rightarrow C^{*}(G / N)$ is the canonical quotient map. A Morita equivalence between twisted coactions $\left(A, G, G / K, \delta_{A}, \tau_{A}\right)$ and $\left(B, G, G / K, \delta_{B}, \tau_{B}\right)$ is an $\left(A, G, \delta_{A}\right)-\left(B, G, \delta_{B}\right)$ Morita equivalence $\left(X, \delta_{X}\right)$ such that

$$
\delta_{X} \mid(x)=\tau_{A} \otimes \operatorname{id}\left(w_{G / K}\right)(x \otimes 1) \tau_{B} \otimes \operatorname{id}\left(w_{G / K}\right)^{*} \quad \text { for } x \in X .
$$

In this case, for any closed normal subgroup $N$ of $G$ contained in $K$, there are natural $A \times_{G / K} G-B \times_{G / K} G$ and $A \times_{G / K} G / N-B \times_{G / K} G / N$ imprimitivity bimodules $X \times_{G / K} G$ and $X \times_{G / K} G / N$, respectively [Ng95, Theorem 3.10], [ER95, Theorem 3.2]. Moreover, there is an action of $K$ on $X \times_{G / K} G$ inducing a Morita equivalence between the dual actions $\left(A \times_{G / K} G, K, \tilde{\delta}_{A}\right)$ and $\left(B \times_{G / K} G, K, \tilde{\delta}_{B}\right)$ 
[ER96, Lemma 4.2], so there is an $A \times_{G / K} G \times{ }_{r} K-B \times_{G / K} G \times{ }_{r} K$ imprimitivity bimodule $X \times_{G / K} G \times{ }_{r} K[\mathrm{Com} 84, \S 6]$.

The next result shows Corollary 3.2 is compatible with Morita equivalence:

Theorem 5.4. If $\left(X, \delta_{X}\right)$ is a Morita equivalence between nondegenerate twisted coactions $\left(A, G, G / K, \delta_{A}, \tau_{A}\right)$ and $\left(B, G, G / K, \delta_{B}, \tau_{B}\right)$, and $N \subset H$ are closed normal subgroups of $G$ contained in $K$ such that Mansfield imprimitivity works for $H$ and one of the coactions, then the cube

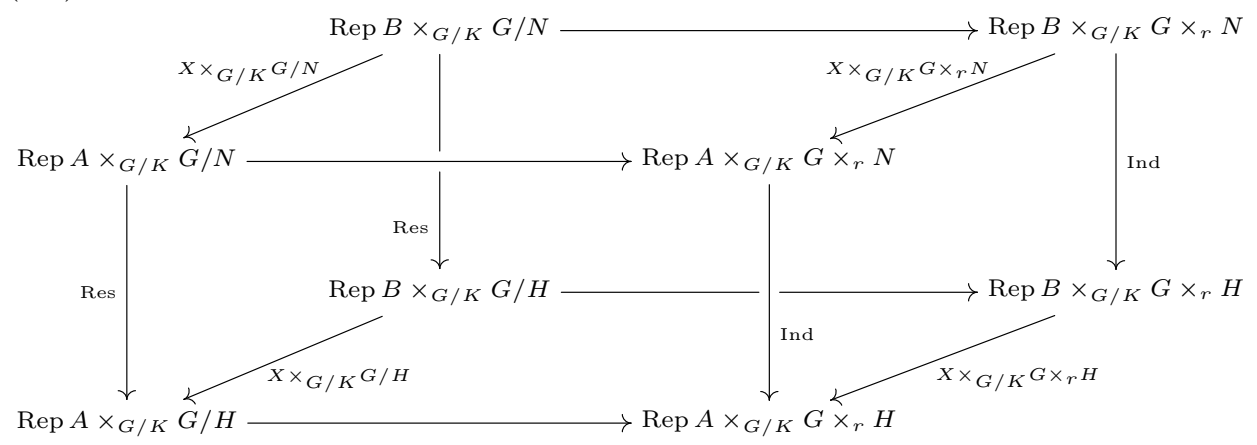

commutes in the usual strong sense.

Proof. First note that by [KQ, Theorem 5.3], if Mansfield imprimitivity works for $H$ and one of the coactions, it works for the other, so the above cube makes sense.

Because all the horizontal arrows are bijections, we need only show commutativity of three of the vertical faces, as well as the top and bottom. The front and back commute by Corollary 3.2.

The bottom face is just the top face with $N$ replaced by $H$; we show the top commutes. The untwisted version (i.e., with $K=G$ )

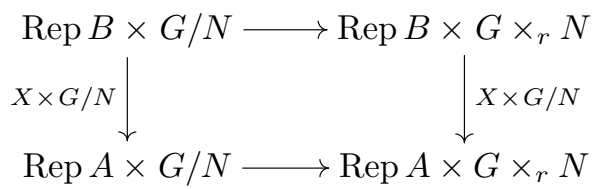

is [ER96, Proposition 4.5]. Even though they use reduced coactions and require $N$ to be amenable, their arguments carry over to our setting since we assume that Mansfield imprimitivity works, as pointed out in [KQ]. So, it only remains to show the ideals in diagram (5.2) match up and appeal to Corollary 2.4. The ideals match up along the top and bottom by [KQ, Theorem 4.4], and along the left side by [ER95, Corollary 3.3], and this is enough, since the top and bottom are Morita equivalences.

For the left face, we use the following general lemma:

Lemma 5.5. Let $\left(X, \delta_{X}\right)$ be a Morita equivalence between coactions $\left(A, G, \delta_{A}\right)$ and $\left(B, G, \delta_{B}\right)$, and let $N$ be a closed normal subgroup of $G$. Then the diagram

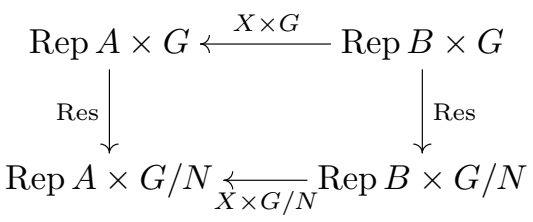

commutes. 
Proof. We aim to apply Lemma 5.3, so we need a nondegenerate imprimitivity bimodule homomorphism

$$
\Phi:_{A \times G / N}(X \times G / N)_{B \times G / N} \rightarrow M\left({ }_{A \times G}(X \times G)_{B \times G}\right)
$$

with

$$
\Phi_{A \times G / N}=j_{A} \times j_{G}^{A} \mid \text { and } \Phi_{B \times G / N}=j_{B} \times j_{G}^{B} \mid .
$$

Let $L(X)$ be the linking algebra for $X$; then there is a coaction $\left(\begin{array}{cc}\delta_{A} & \delta_{X} \\ \delta_{\tilde{X}} & \delta_{B}\end{array}\right)$ of $G$ on $L(X)$. By [ER95, Appendix], we have

$$
L(X) \times G / N=L(X \times G / N)
$$

and

$$
M(L(X) \times G))=\left(\begin{array}{ll}
M(A \times G) & M(X \times G) \\
M(\tilde{X} \times G) & M(B \times G)
\end{array}\right) .
$$

By Lemma 5.2, the nondegenerate homomorphism

$$
j_{L(X)} \times j_{G}^{L(X)} \mid: L(X) \times G / N \rightarrow M(L(X) \times G)
$$

restricts on the corners to a nondegenerate imprimitivity bimodule homomorphism

$$
\Phi:_{A \times G / N}(X \times G / N)_{B \times G / N} \rightarrow M\left({ }_{A \times G}(X \times G)_{B \times G}\right) .
$$

Since the restrictions of $j_{L(X)} \times j_{G}^{L(X)} \mid$ to the diagonal corners $A \times G / N$ and $B \times G / N$ agree with $j_{A} \times j_{G}^{A} \mid$ and $j_{B} \times j_{G}^{B} \mid$, respectively, we are done.

Returning to the proof of Theorem 5.4, the untwisted version of the left-hand face of diagram (5.1) follows from Lemma 5.5. We need only show that the twisting ideals in each crossed product in diagram (5.3) match up, and apply Corollary 2.4. That the ideals match up across the horizontal arrows is shown in [ER95, Corollary 3.3]. That the ideals match up along the vertical arrows follows from the proof of Corollary 3.2. This completes the proof of Theorem 5.4

In the proof of Theorem 5.4, we were able to deduce commutativity of the right face of (5.1) from the other five faces. This is a special case of a general "IndInd" diagram for actions which is related to [Ech94b, Theorem 3] and [Kal94, Proposition 4.1.2].

We now show that Corollary 4.3 is compatible with Morita equivalence:

Theorem 5.6. If $\left(X, \delta_{X}\right)$ is a Morita equivalence between nondegenerate twisted coactions $\left(A, G, G / K, \delta_{A}, \tau_{A}\right)$ and $\left(B, G, G / K, \delta_{B}, \tau_{B}\right)$, and $N \subset H$ are closed normal subgroups of $G$ contained in $K$ such that Mansfield imprimitivity works for $H$ 
and one of the coactions, then the cube

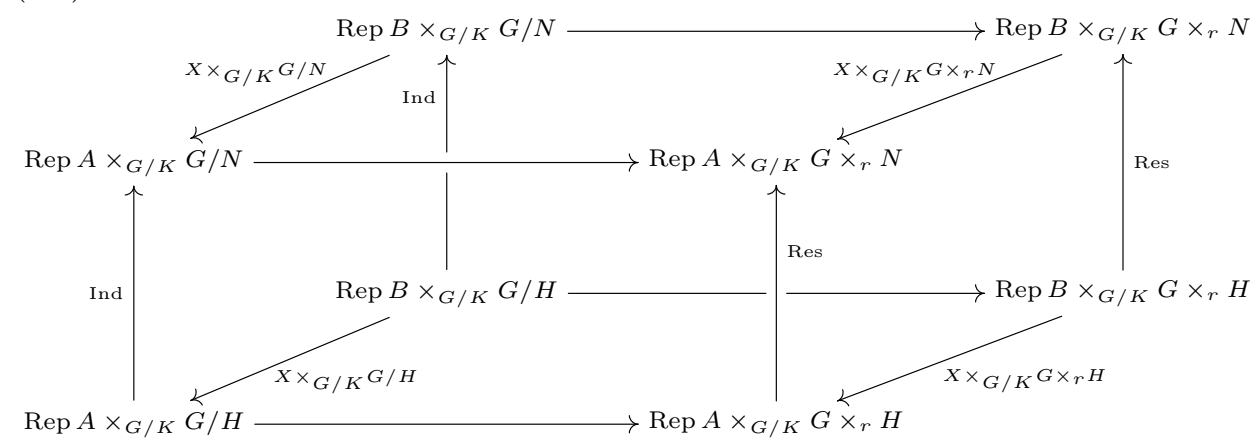

commutes in the usual strong sense.

Proof. The front and back faces commute by Corollary 4.3, and the top and bottom faces are the same as in (5.1), so it suffices to show commutativity of the right face. For this we use the following lemma:

Lemma 5.7. Let $(Y, \gamma)$ be a Morita equivalence between actions $(C, H, \alpha)$ and $(D, H, \beta)$, and let $N$ be a closed subgroup of $H$. Then the diagram

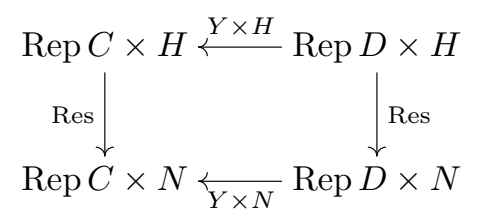

commutes.

Proof. We aim to apply Lemma 5.3, so we need a nondegenerate imprimitivity bimodule homomorphism

$$
\Phi:_{C \times N}(Y \times N)_{D \times N} \rightarrow M\left(C \times H(Y \times H)_{D \times H}\right)
$$

with

$$
\Phi_{C \times N}=i_{C} \times\left. i_{H}^{C}\right|_{N} \quad \text { and } \quad \Phi_{D \times N}=i_{D} \times\left. i_{H}^{D}\right|_{N}
$$

Let $L(Y)$ be the linking algebra for $Y$; then there is an action $\left(\begin{array}{ll}\alpha & \gamma \\ \tilde{\gamma} & \beta\end{array}\right)$ of $H$ on $L(Y)$. By [ER95, Appendix], we have

$$
L(Y) \times N=L(Y \times N)
$$

and

$$
M(L(Y) \times H))=\left(\begin{array}{ll}
M(C \times H) & M(Y \times H) \\
M(\tilde{Y} \times H) & M(D \times H)
\end{array}\right) .
$$

By Lemma 5.2, the nondegenerate homomorphism

$$
i_{L(Y)} \times\left. i_{H}^{L(Y)}\right|_{N}: L(Y) \times N \rightarrow M(L(Y) \times H)
$$

restricts on the corners to a nondegenerate imprimitivity bimodule homomorphism

$$
\Phi:_{C \times N}(Y \times N)_{D \times N} \rightarrow M\left(C \times H(Y \times H)_{D \times H}\right) .
$$

Since the restrictions of $i_{L(Y)} \times\left. i_{H}^{L(Y)}\right|_{N}$ to the diagonal corners $C \times N$ and $D \times N$ agree with $i_{C} \times\left. i_{H}^{C}\right|_{N}$ and $i_{D} \times\left. i_{H}^{D}\right|_{N}$, respectively, we are done. 
Returning to the proof of the right-hand face of diagram (5.4), we need only show that the kernels of the regular representations of each crossed product in diagram (5.5) match up, and apply Corollary 2.4. That the kernels match up across the horizontal arrows is shown in [Com84, §6]. The kernels match up along the vertical arrows because the regular representation of $N$ is quasi-equivalent to the regular representation of $H$ restricted to $N$. Alternatively, we can argue as follows: we must show that

$$
\operatorname{Res}_{C \times H}^{C \times N} \operatorname{Ind}_{C}^{C \times H}\{0\}=\operatorname{Ind}_{C}^{C \times N}\{0\} .
$$

By Green induction in stages,

$$
\operatorname{Ind}_{C}^{C \times H}=\operatorname{Ind}_{C \times N}^{C \times H} \circ \operatorname{Ind}_{C}^{C \times N} .
$$

Also, [Gre78, Proposition 11] implies that

$$
\operatorname{Res}_{C \times H}^{C \times N} \operatorname{Ind}_{C \times N}^{C \times H} K=K
$$

for any $H$-invariant ideal $K$ of $C \times N$. Since for $N$ normal, $\operatorname{Ind}_{C}^{C \times N}\{0\}$ is $H$ invariant, this gives us

$$
\begin{aligned}
\operatorname{Res}_{C \times H}^{C \times N} \operatorname{Ind}_{C}^{C \times H}\{0\} & =\operatorname{Res}_{C \times H}^{C \times N} \operatorname{Ind}_{C \times N}^{C \times H} \operatorname{Ind}_{C}^{C \times N}\{0\} \\
& =\operatorname{Ind}_{C}^{C \times N}\{0\} .
\end{aligned}
$$

This completes the proof of Theorem 5.6.

Remark 5.8. By [KQ, Proposition 2.3], Morita equivalence respects nondegeneracy of coactions. Hence, in Theorems 5.4 and 5.6, one coaction is in fact nondegenerate if and only if the other one is.

This time, we were able to deduce commutativity of the left face of (5.4) from the other five faces. Written in terms of tensor products,

$$
\begin{aligned}
& Z_{G / H}^{G / N}(A) \otimes_{A \times_{G / K} G / H}\left(X \times_{G / K} G / H\right) \\
& \quad \cong\left(X \times_{G / K} G / N\right) \otimes_{B \times_{G / K} G / N} Z_{G / H}^{G / N}(B),
\end{aligned}
$$

where we have used self-explanatory notation to distinguish the bimodules for $A$ and $B$. For $N=\{e\}$, the commutativity of this left face, in the weak sense, follows from [ER96, Theorem 4.4], as in the discussion preceding [ER96, Theorem 4.7] one could presumably get a direct proof of (5.6) by strengthening the arguments of [ER96] and replacing $G$ by $G / N$.

We turn to inflation of coactions: if $K$ is a closed normal subgroup of $G$ and $(A, K, \epsilon)$ is a coaction, composing with the natural embedding of $A \otimes C^{*}(K)$ in $M\left(A \otimes C^{*}(G)\right)$ gives a coaction $(A, G, \operatorname{Inf} \epsilon)$, called an inflated coaction. Inf $\epsilon$ is trivially twisted over $G / K$ by $f \mapsto f(e) 1$, and [PR94, Example 2.14] gives a natural isomorphism of $A \times \times_{\operatorname{Inf} \epsilon, G / K} G$ onto $A \times{ }_{\epsilon} K$ which takes $k_{A}(a) k_{G}(f)$ to $j_{A}(a) j_{K}\left(\left.f\right|_{K}\right)$.

The next two theorems show Corollaries 3.2 and 4.3 are compatible with inflation:

Theorem 5.9. If $N \subset H$ are closed normal subgroups of $G$ contained in $K$, and $(A, K, \epsilon)$ is a nondegenerate coaction such that Mansfield imprimitivity works for 
$H$ and $\epsilon$, then the cube

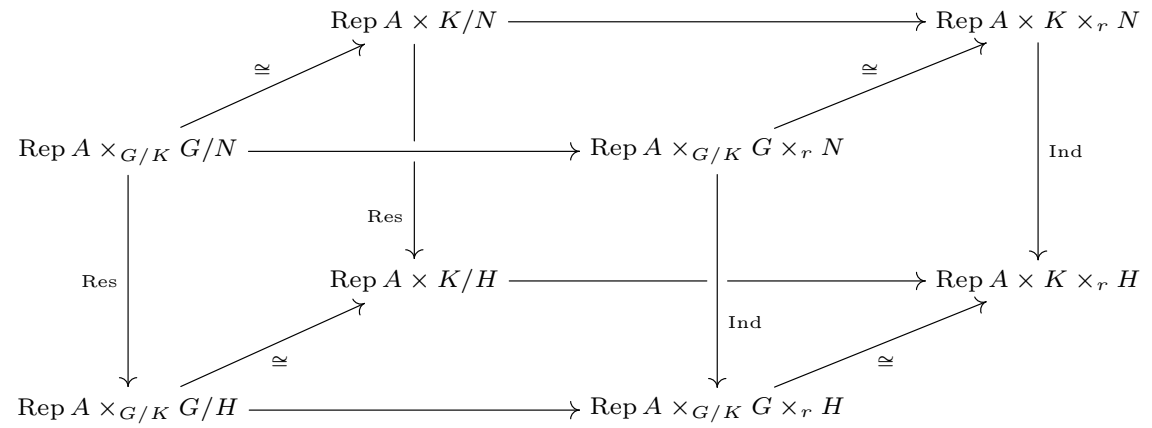

commutes in the usual strong sense.

Proof. First, by [KQ, Theorem 5.4], Mansfield imprimitivity works for $H$ and the (nondegenerate) inflated coaction $(A, G, \operatorname{Inf} \epsilon)$, so the above diagram makes sense.

The front and back faces commute by Theorem 3.1 and Corollary 3.2 .

We show the top commutes; the bottom is essentially the same. [ER96, Proposition 4.8 and Remark 4.9] give an imprimitivity bimodule homomorphism

$$
\left(\Phi_{N}, \Phi_{Y}, \Phi_{G / N}\right): Y_{G / N}^{G} \rightarrow Y_{K / N}^{K} .
$$

Hence, Lemma 5.3 gives a commutative diagram

$$
\begin{gathered}
\operatorname{Rep} A \times G / N \stackrel{Y_{G / N}^{G}}{\longrightarrow} \operatorname{Rep} A \times G \times{ }_{r} N \\
\Phi_{G / N}^{*} \uparrow \\
\operatorname{Rep} A \times K / N \underset{Y_{K / N}^{K}}{\longrightarrow} \operatorname{Rep} A \times K \times_{r}^{*} N .
\end{gathered}
$$

To pass from (5.8) to the top of (5.7), we need to show the appropriate ideals $I_{\tau}^{N}$ in $A \times G / N, X_{\{e\}}^{N}$-Ind $I_{\tau}$ in $A \times G \times{ }_{r} N$, and $\{0\}$ in both $A \times K / N$ and $A \times K \times{ }_{r} N$ match up. The ideals match up along the top (by [KQ, Theorem 4.4]), bottom (trivially), and left (since $\operatorname{ker} \Phi_{G / N}=I_{\tau}^{N}$ ), and this is enough.

Finally, for the left face of (5.7), the diagram

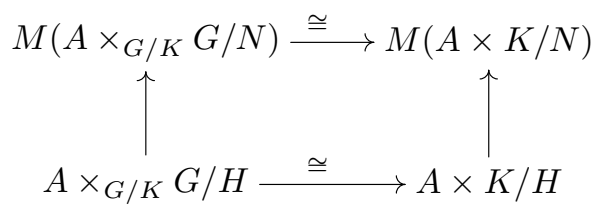

of nondegenerate homomorphisms commutes, since the diagram

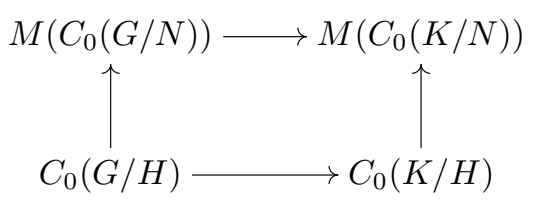


does. Hence, the required diagram

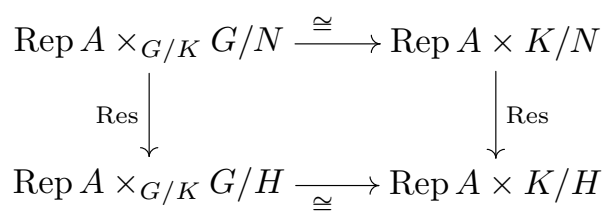

of right-Hilbert bimodules commutes as well.

Theorem 5.10. If $N \subset H$ are closed normal subgroups of $G$ contained in $K$, and $(A, K, \epsilon)$ is a nondegenerate coaction such that Mansfield imprimitivity works for $H$ and $\epsilon$, then the cube

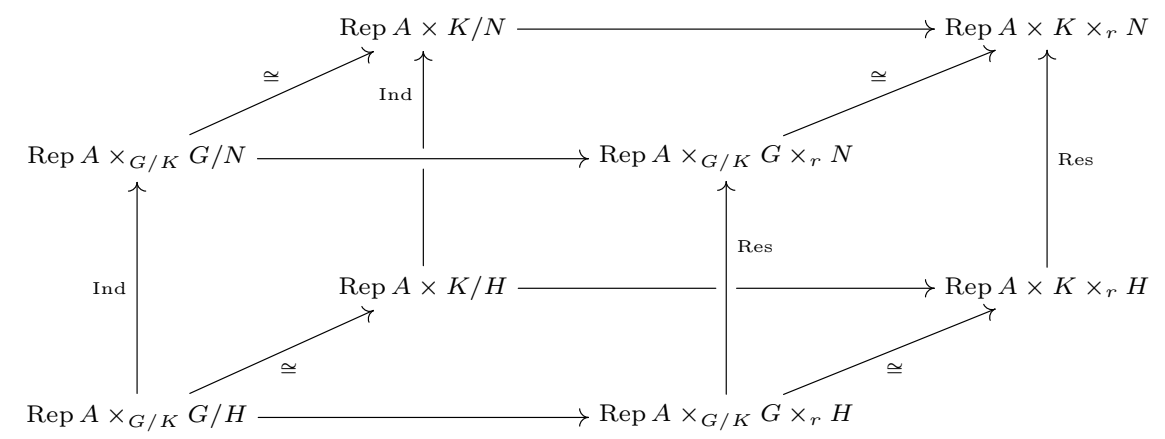

commutes in the usual strong sense.

Proof. The front and back faces commute by Theorem 4.1 and Corollary 4.3, and the top and bottom faces are the same as in (5.7). Since the horizontal maps in the right face come from equivariant isomorphisms, the commutativity of this face follows from Lemma 5.7 as in the proof of Theorem 5.6.

Remark 5.11. By [KQ, Proposition 2.1], in Theorems 5.9 and 5.10, Inf $\epsilon$ is in fact nondegenerate if and only if $\epsilon$ is. Moreover, when the coactions are nondegenerate, Mansfield imprimitivity works for $H$ and $\operatorname{Inf} \epsilon$ if and only if it works for $H$ and $\epsilon$.

As before, we get commutativity of a sixth face of diagrams (5.7) and (5.9) from the other five faces. The left face of (5.9) is new; commutativity in the weak sense can be deduced from [ER96, Theorem 4.7].

Finally, we show that our Res-Ind duality is compatible with the stabilization trick of [ER96], as adapted to full coactions in [KQ]. Let $(A, G, G / K, \delta, \tau)$ be a nondegenerate twisted coaction such that Mansfield imprimitivity works for $\delta$ and $K$ itself; equivalently, such that $\delta$ is normal [KQ, Lemma 3.6]. Then Mansfield imprimitivity works for $\delta$ and any closed normal subgroup of $G$ contained in $K$ by $[\mathrm{KQ}$, Theorem 5.2] (or by [KQ, Lemma 3.2], since $\delta$ is normal). [ER96, Theorem 3.1] shows that the twisted coaction $(A, G, G / K, \delta, \tau)$ is Morita equivalent to the (nondegenerate) inflated twisted coaction $\left(A \times_{G / K} G \times{ }_{r} K, G, G / K, \operatorname{Inf}(\widehat{\widetilde{\delta}})^{\mathrm{n}}, 1\right)$, so by [KQ, Theorems 5.3 and 5.4], Mansfield imprimitivity works for $(\widetilde{\widetilde{\delta}})^{\mathrm{n}}$ and any closed normal subgroup of $G$ contained in $K$. Thus we can chain together Theorems 5.4 and 5.9, and similarly Theorems 5.6 and 5.10, to obtain: 
Theorem 5.12. Let $(A, G, G / K, \delta, \tau)$ be a nondegenerate normal twisted coaction, and let $N \subset H$ be closed normal subgroups of $G$ contained in $K$. Further let $B=A \times_{G / K} G \times{ }_{r} K$, which carries the double dual coaction of $K$. Then the cubes

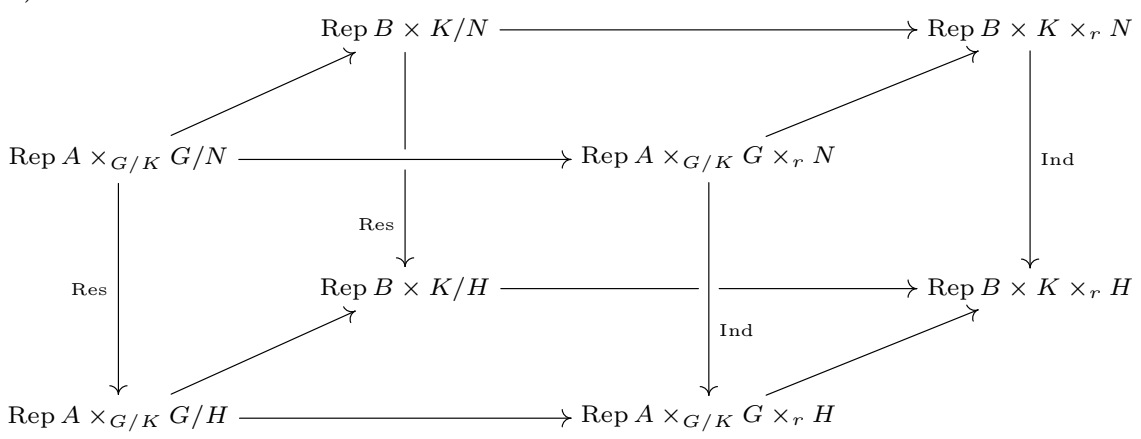

and

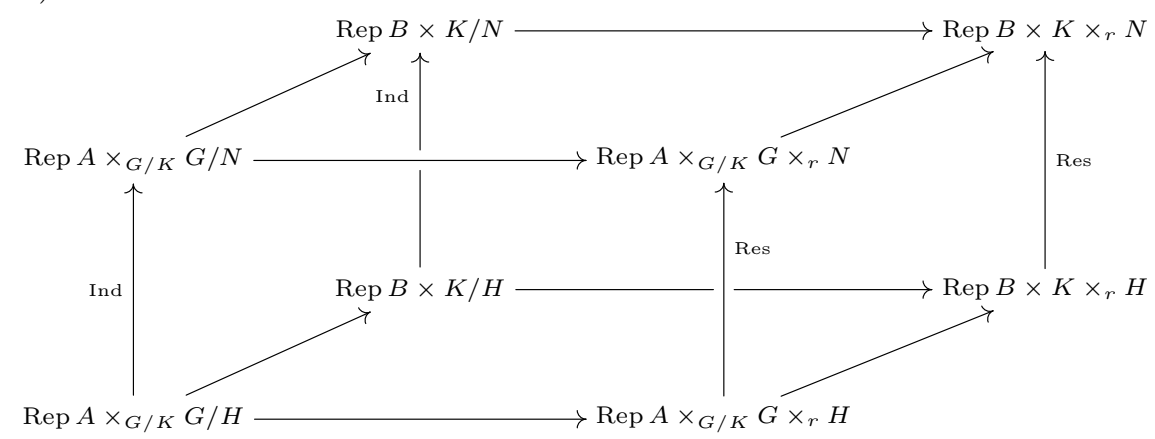

both commute in the usual strong sense.

Again, the left face of (5.11) is new; the weak form can be deduced from [ER96, Theorem 4.7].

\section{Ind, Res, Ex, SuB}

We will now use our techniques to extend to nonamenable groups some results of Gootman and Lazar [GL89, §3] on restriction and induction of ideals in crossed products by coactions of amenable groups; Nilsen [Nil96] has recently proved similar results using representation-theoretic methods. With some additional effort, we could further generalize to situations involving induction and restriction from crossed products by quotients, but this would complicate matters substantially.

Recall from [Gre78, Proposition 9] that a nondegenerate homomorphism $\varphi: A \rightarrow$ $M(B)$ gives rise to maps $\operatorname{Res}_{\varphi}=\varphi^{*}: \mathcal{I}(B) \rightarrow \mathcal{I}(A)$ and $\operatorname{Ex}_{\varphi}=\varphi_{*}: \mathcal{I}(A) \rightarrow \mathcal{I}(B)$ between spaces of ideals: by definition, $\operatorname{Res}_{\varphi}(J)=\{a \in A \mid \varphi(a) B \subset J\}$, and $\operatorname{Ex}_{\varphi}(I)$ is the ideal generated by $\varphi(I) B$. If in addition $X$ is a $B-C$ imprimitivity bimodule, then there is also an induction $\operatorname{map} \operatorname{Ind}_{\varphi}:=\operatorname{Res}_{\varphi} \circ X$-Ind $: \mathcal{I}(C) \rightarrow \mathcal{I}(A)$. Green defines an analogous map $\operatorname{Sub}_{\varphi}: \mathcal{I}(A) \rightarrow \mathcal{I}(C)$ by $\operatorname{Sub}_{\varphi}:=\tilde{X}$-Ind $\circ \operatorname{Ex}_{\varphi}$. The various maps are related as follows:

Proposition 6.1 ([Gre78, Proposition 9(i)]). Fix ideals $I$ of $A, J$ of $B$, and $K$ of C. Then: 
(i) $\operatorname{Res} \operatorname{Ex} I \supset I$; Ind $\operatorname{Sub} I \supset I$;

(ii) $\operatorname{Ex} \operatorname{Res} J \subset J$; Sub Ind $K \subset K$;

(iii) Ind, Res, Sub and Ex are order-preserving;

(iv) Ind and Res preserve arbitrary intersections;

(v) Ex Res Ex $I=\operatorname{Ex} I$; Sub Ind Sub $I=\operatorname{Sub} I$;

(vi) Ex $I$ is the smallest ideal $J^{\prime}$ of $B$ such that Res $J^{\prime} \supset I$;

(vii) Sub $I$ is the smallest ideal $K^{\prime}$ of $C$ such that Ind $K^{\prime} \supset I$.

Now consider an action $(B, G, \alpha)$. The usual restriction map $\operatorname{Res}_{B \times G}^{B}$ is the map $\operatorname{Res}_{i_{B}}$ associated to the inclusion $i_{B}: B \rightarrow M(B \times G)$, and $\operatorname{Ind}_{B}^{B \times G}$ is the map Ind $_{j_{B \times G}}$ associated to $j_{B \times G}: B \times G \rightarrow M(B \times G \times G)$ and Green's $B \times G \times G-$ $B$ imprimitivity bimodule (where we have identified Green's imprimitivity algebra $C_{0}(G, B) \times G$ with the isomorphic co-crossed product $\left.B \times G \times G\right)$. We now have in addition $\operatorname{Ex}_{B}^{B \times G}:=\operatorname{Ex}_{i_{B}}$ and $\operatorname{Sub}_{B \times G}^{B}:=\operatorname{Sub}_{j_{B \times G}}$. Green proves:

Proposition 6.2 ([Gre78, Proposition 11]). Let $(B, G, \alpha)$ be an action, and fix $I \in$ $\mathcal{I}(B)$ and $J \in \mathcal{I}(B \times G)$. Then:

(i) Res $J$ and Sub $J$ are $G$-invariant;

(ii) Res Ind $I$ is the largest $G$-invariant ideal of $B$ contained in $I$;

(iii) $I$ is $G$-invariant if and only if $\operatorname{Res} I n d ~ I=I$;

(iv) Ind Res Ind $I=$ Ind $I$.

We will need the analogue of this result for reduced crossed products. By definition $\operatorname{Res}_{B \times_{r} G}^{B}$ is the map $\operatorname{Res}_{\rho \circ i_{B}}$ associated to the composition of $i_{B}$ with the quotient map $\rho: B \times G \rightarrow B \times{ }_{r} G$, and we can define $\operatorname{Ex}_{B}^{B \times_{r} G}:=\operatorname{Ex}_{\rho \circ i_{B}}$. Unravelling the definitions gives the relations

$$
\operatorname{Res}_{B \times_{r} G}^{B}(J)=\operatorname{Res}_{B \times G}^{B}\left(\rho^{-1}(J)\right) \text { and } \operatorname{Ex}_{B}^{B \times{ }_{r} G}(I)=\rho\left(\operatorname{Ex}_{B}^{B \times G}(I)\right) .
$$

Now because $(B \times G) \times G \cong\left(B \times{ }_{r} G\right) \times G$ [Rae92, Proposition 3.2 and Theorem 4.1], we can view Green's bimodule as a $B \times{ }_{r} G \times G-B$ imprimitivity bimodule, which together with the embedding $j_{B \times{ }_{r} G}: B \times{ }_{r} G \rightarrow M\left(B \times{ }_{r} G \times G\right)$ gives us $\operatorname{maps}_{\operatorname{Ind}_{B}^{B} \times_{r} G}:=\operatorname{Ind}_{j_{B \times r} G}$ and $\operatorname{Sub}_{B \times_{r} G}^{B}:=\operatorname{Sub}_{j_{B \times r} G}$. The commutativity (by construction of the "reduction map" in [Rae92, Theorem 4.1]) of the diagram

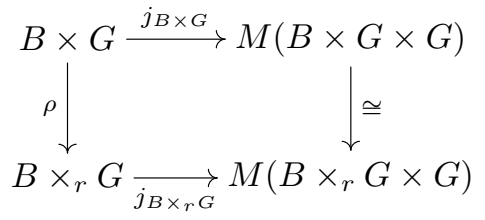

then implies that

$$
\operatorname{Ind}_{B}^{B \times{ }_{r} G}(I)=\rho\left(\operatorname{Ind}_{B}^{B \times G}(I)\right) \text { and } \operatorname{Sub}_{B \times_{r} G}^{B}(J)=\operatorname{Sub}_{B \times G}^{B}\left(\rho^{-1}(J)\right) .
$$

Now for any ideal $J$ of $B$, $\operatorname{ker} \rho=\operatorname{Ind}_{B}^{B \times G}\{0\} \subset \operatorname{Ind}_{B}^{B \times G} J$, so $\operatorname{Ind}_{B}^{B \times G} J \vee \operatorname{ker} \rho=$ $\operatorname{Ind}_{B}^{B \times G} J$. Thus,

$$
\begin{aligned}
\operatorname{Res}_{B \times{ }_{r} G}^{B} \operatorname{Ind}_{B}^{B \times{ }_{r} G} J & =\operatorname{Res}_{B \times G}^{B} \rho^{-1} \rho\left(\operatorname{Ind}_{B}^{B \times G} J\right) \\
& =\operatorname{Res}_{B \times G}^{B}\left(\operatorname{Ind}_{B}^{B \times G} J \vee \operatorname{ker} \rho\right) \\
& =\operatorname{Res}_{B \times G}^{B} \operatorname{Ind}_{B}^{B \times G} J,
\end{aligned}
$$


and we can deduce that Proposition 6.2 carries over to reduced crossed products.

We now consider a nondegenerate normal coaction $(A, G, \delta)$. The canonical map $j_{A}: A \rightarrow M(A \times G)$ gives maps $\operatorname{Res}_{A \times G}^{A}=\operatorname{Res}_{j_{A}}$ and $\operatorname{Ex}_{A}^{A \times G}:=\operatorname{Ex}_{j_{A}}$. Similarly, $i_{A \times G}: A \times G \rightarrow M\left(A \times G \times{ }_{r} G\right)$ and the $A \times G \times{ }_{r} G-A$ imprimitivity bimodule $Y$ from Mansfield's imprimitivity theorem give maps $\operatorname{Ind}_{A}^{A \times G}=\operatorname{Ind}_{i_{A \times G}}$ and $\operatorname{Sub}_{A \times G}^{A}:=\operatorname{Sub}_{i_{A \times G}}=\tilde{Y}$-Ind $\circ \operatorname{Ex}_{i_{A \times G}}$. In this situation, Theorem 3.1 in the case $N=\{e\}$ and $H=G$ gives us the commutativity of the following diagram:

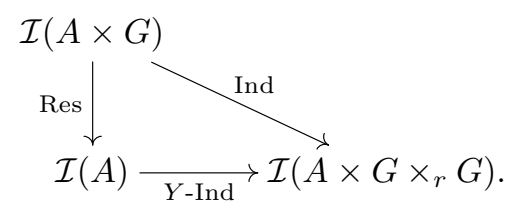

From this we can deduce the analogous Ex-Sub duality:

Theorem 6.3. Let $(A, G, \delta)$ be a nondegenerate normal coaction. Then we have a commutative diagram:

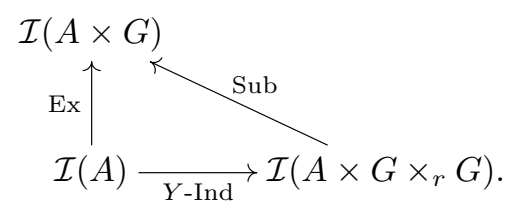

Proof. Fix $I \in \mathcal{I}(A)$. By Proposition 6.1(vi), Ex $I$ is the smallest ideal $J$ of $A \times G$ such that Res $J \supset I$; equivalently, such that $Y$-Ind(Res $J) \supset Y$-Ind $I$. Hence, by (6.2), Ex $I$ is the smallest ideal $J$ of $A \times G$ such that Ind $J \supset Y$-Ind $I$. Using Proposition 6.1(vii), this implies that

$$
\operatorname{Ex} I=\operatorname{Sub}(Y-\operatorname{Ind} I)
$$

so the diagram commutes.

Remark 6.4. When there are no subgroups or twists, Theorem 4.1 reduces to the definition of $\operatorname{Ind}_{A}^{A \times G}$. When there is either a twist or an intermediate subgroup, there will be an analogous Sub-Ex duality dual to (6.3) which reduces in the absence of the twist or subgroup to the definition of Sub. So our whole discussion has been considerably simplified by the decision to ignore twists and intermediate subgroups. Nevertheless, we believe that our techniques will still work in full generality.

We are now ready to generalize the results of Gootman and Lazar. Let $(A, G, \delta)$ be a nondegenerate normal coaction. As we pointed out in the introduction, Mansfield imprimitivity always involves reduced crossed products, so in what follows all the maps Res, Ex, etc. involving crossed products by actions are the reduced versions $\operatorname{Res}_{B \times{ }_{r} G}^{B}, \operatorname{Ex}_{B}^{B \times{ }_{r} G}$, etc. we discussed above. Thus we hope that no confusion will arise if we drop all the subscripts and superscripts.

Adapting [ER96, Definition 2.4] to full coactions, we say that an ideal $I$ of $A$ is $G$-invariant if

$$
I=\operatorname{ker}\left(q_{I} \otimes \lambda\right) \circ \delta
$$

where $q_{I}: A \rightarrow A / I$ is the quotient map and $\lambda: C^{*}(G) \rightarrow C_{r}^{*}(G)$ is the left regular representation. We do not know if this implies that $\delta$ restricts to a coaction on $I$ (unless $G$ is amenable), but we do get a coaction $\delta_{A / I}$ of $G$ on $A / I$. Since we will not need this fact here, we omit the proof. 
Let ${ }^{G} \mathcal{I}(A)$ denote the $G$-invariant ideals of $A$.

Lemma 6.5. Let $(A, G, \delta)$ be a nondegenerate normal coaction.

(i) An ideal I of $A$ is $G$-invariant if and only if

$$
I=\operatorname{Res} \text { Ind } I \text {. }
$$

(ii) Ind is injective when restricted to ${ }^{G} \mathcal{I}(A)$, and $\operatorname{Res} \mathcal{I}(A \times G)$ contains ${ }^{G} \mathcal{I}(A)$.

Proof. Part (ii) is immediate from (i). To see (i), note that [Man91, Proposition 21] implies that Ind $q_{I} \sim\left(q_{I} \otimes \lambda\right) \circ \delta \times(1 \otimes M)$; hence Res Ind $q_{I} \sim\left(q_{I} \otimes \lambda\right) \circ \delta$, and we have:

$$
\begin{aligned}
\operatorname{ker}\left(q_{I} \otimes \lambda\right) \circ \delta & =\operatorname{ker}\left(\operatorname{Res} \operatorname{Ind} q_{I}\right) \\
& =\operatorname{Res} \operatorname{Ind}\left(\operatorname{ker} q_{I}\right) \\
& =\operatorname{Res} \operatorname{Ind} I . \quad \square
\end{aligned}
$$

Green shows [Gre78, Proposition 13] that for an action $(B, G, \alpha)$ of an amenable group $G, \mathrm{Ex}=$ Ind on ${ }^{G} \mathcal{I}(B)$. This is definitely not true for non-amenable $G$ : $\operatorname{Ex}\{0\}=\{0\}$, while $\operatorname{Ind}\{0\}=\{0\}$ if and only if $B \times G=B \times{ }_{r} G$. For all we know, even if we pass to reduced crossed products, Ex and Ind can be different on ${ }^{G} \mathcal{I}(B)$. Even for trivial actions, the question reduces to the unsolved problem of whether there exists a locally compact group $G$ for which $C_{r}^{*}(G)$ is not exact.

Coactions behave like actions of abelian groups, so the following generalization of [GL89, Proposition 3.14(iii)] is not surprising:

Lemma 6.6. Let $(A, G, \delta)$ be a nondegenerate normal coaction, and let $I \in{ }^{G} \mathcal{I}(A)$. Then

$$
\operatorname{Ex} I=\operatorname{Ind} I \text {, }
$$

and Ex is injective when restricted to ${ }^{G} \mathcal{I}(A)$.

Proof. Using dualities (6.2) and (6.3), together with Proposition 6.2 (i) and (iv), we have:

$$
\begin{aligned}
\text { Ind Res Ex } I & =(\operatorname{Res} Y \text {-Ind })(\tilde{Y} \text {-Ind Ind })(\operatorname{Sub} Y \text {-Ind } I) \\
& =\operatorname{Res} \text { Ind Sub } Y \text {-Ind } I \\
& =\operatorname{Sub} Y \text {-Ind } I \\
& =\operatorname{Ex} I
\end{aligned}
$$

so since Ind is order-preserving and Res Ex is increasing, we have

$$
\text { Ind } I \subset \operatorname{Ind} \operatorname{Res} \operatorname{Ex} I=\operatorname{Ex} I \text {. }
$$

On the other hand, by invariance we have $I=\operatorname{Res} I n d ~ I$ (Proposition 6.5(i)), hence

$$
\text { Ex } I=\text { Ex Res Ind } I \subset \text { Ind } I \text {, }
$$

since Ex Res is decreasing. The last statement of the lemma is immediate from Lemma 6.5(ii).

We do not know how to prove the companion result for Sub and Res (generalizing [GL89, Proposition 3.14(iv)]); it certainly doesn't follow from [Gre78] and duality as in the proof of Lemma 6.6.

We next show that the maps Res, Ind and Ex produce invariant ideals, extending parts of [GL89, Propositions 3.14(i) and 3.15(i)] to the non-amenable case. 
Proposition 6.7. Let $(A, G, \delta)$ be a nondegenerate normal coaction, and fix $I \in$ $\mathcal{I}(A)$ and $J \in \mathcal{I}(A \times G)$. Then:

(i) Ind $I$ and $\operatorname{Ex} I$ are in ${ }^{G} \mathcal{I}(A \times G)$;

(ii) $\operatorname{Res} J$ is in ${ }^{G} \mathcal{I}(A)$.

Proof. By definition,

$$
\text { Ind } I=\operatorname{Res} Y \text {-Ind } I \text {, }
$$

and by the Ex-Sub duality (6.3),

$$
\operatorname{Ex} I=\operatorname{Sub} Y \text {-Ind } I,
$$

so (i) follows from Proposition 6.2(i).

For (ii), Proposition 6.2(iv) and repeated use of Res-Ind duality (6.2) gives

$$
\begin{aligned}
\text { Res Ind Res } J & =(\tilde{Y} \text {-Ind Ind })(\operatorname{Res} Y \text {-Ind })(\tilde{Y} \text {-Ind Ind } J) \\
& =\tilde{Y} \text {-Ind Ind Res Ind } J \\
& =\tilde{Y} \text {-Ind Ind } J \\
& =\operatorname{Res} J
\end{aligned}
$$

so $\operatorname{Res} J$ is invariant by Lemma 6.5 .

The following proposition generalizes [GL89, Proposition 3.14(v)].

Proposition 6.8. Let $(A, G, \delta)$ be a nondegenerate normal coaction, and fix $I \in$ $\mathcal{I}(A)$ and $J \in \mathcal{I}(A \times G)$. Then:

$$
\text { Ind Res Ind } I=\operatorname{Ind} I \text { and } \quad \operatorname{Res} I n d \operatorname{Res} J=\operatorname{Res} J \text {. }
$$

Proof. Since Ind $I$ is invariant (Proposition 6.7(i)), we have

$$
\text { Ind } I=\text { Res Ind Ind } I=\text { Ind Res Ind } I
$$

by Proposition 6.2(iii) and duality.

Since Res $J$ is invariant (Proposition 6.7(ii)), we have

$$
\text { Res Ind Res } J=\operatorname{Res} J
$$

using Proposition 6.5(i).

The following lemma generalizes part of [GL89, Lemma 3.12], which relies explicitly on the amenability of $G$.

Lemma 6.9. Let $(A, G, \delta)$ be a nondegenerate normal coaction. Then ${ }^{G} \mathcal{I}(A)$ is closed under arbitrary intersections.

Proof. ${ }^{G} \mathcal{I}(A) \subset \operatorname{Res} \mathcal{I}(A \times G)$ (Lemma 6.5) and Res preserves arbitrary intersections; the lemma follows.

Our final result generalizes part of [GL89, Proposition 3.15, (iii) and (iv)]:

Proposition 6.10. Let $(A, G, \delta)$ be a nondegenerate normal coaction, and fix $I \in$ $\mathcal{I}(A)$ and $J \in \mathcal{I}(A \times G)$. Then:

(i) Res Ex $I$ is the smallest $G$-invariant ideal of $A$ containing I;

(ii) Ind Res $J$ is the largest $G$-invariant ideal of $A \times G$ contained in $J$. 
Proof. (i) Let $K$ be the intersection of all invariant ideals of $A$ containing $I$; since ${ }^{G} \mathcal{I}(A)$ is closed under intersections (Lemma 6.9), $K$ is invariant and hence is the smallest such ideal of $A$. Now Res Ex $I$ is invariant (Proposition 6.7(ii)), and $\operatorname{Res} \operatorname{Ex} I \supset I$ because Res Ex is increasing; so we get

$$
I \subset K \subset \operatorname{Res} \operatorname{Ex} I \text {. }
$$

Taking Ex and using Proposition 6.1, we have

$$
\operatorname{Ex} I \subset \operatorname{Ex} K \subset \operatorname{Ex} \operatorname{Res} \operatorname{Ex} I=\operatorname{Ex} I,
$$

so equality holds throughout. In particular,

$$
\operatorname{Ex} K=\operatorname{Ex} \operatorname{Res} \operatorname{Ex} I
$$

since Ex is injective on invariant ideals (Lemma 6.6), we get

$$
\operatorname{Res} \operatorname{Ex} I=K \text {. }
$$

(ii) By the Res-Ind duality (6.2),

$$
\text { Ind Res } J=(\operatorname{Res} Y \text {-Ind })(\tilde{Y} \text {-Ind Ind } J)=\operatorname{Res} \text { Ind } J,
$$

which is the largest $G$-invariant ideal of $A \times G$ contained in $J$, by Proposition 6.2(ii).

\section{REFERENCES}

[BS89] S. Baaj and G. Skandalis. $C^{*}$-algèbres de Hopf et théorie de Kasparov équivariante. K-Theory, 2:683-721, 1989. MR 90j:46061

[Bui94] H. H. Bui. Morita equivalence of twisted crossed products by coactions. J. Funct. Anal., 123:59-98, 1994. MR 95g:46121

[Bui95] H. H. Bui. Full coactions on Hilbert $C^{*}$-modules. J. Austral. Math. Soc. (Ser. A), 59:409420, 1995. MR 96j:46067

[Com84] F. Combes. Crossed products and Morita equivalence. Proc. London Math. Soc., 49:289306, 1984. MR 86c:46081

[Ech] S. Echterhoff. Crossed products with continuous trace. Mem. Amer. Math. Soc. to appear. CMP 96:07

[Ech94a] S. Echterhoff. Duality of induction and restriction for abelian twisted covariant systems. Math. Proc. Camb. Phil. Soc., 116:301 - 315, 1994. MR 96a:46119

[Ech94b] S. Echterhoff. Morita equivalent twisted actions and a new version of the Packer-Raeburn stabilization trick. J. London Math. Soc., 50(2):170-186, 1994. MR 96a:46118

[EKR95] S. Echterhoff, S. Kaliszewski, and I. Raeburn. preprint 1996.

[ER95] S. Echterhoff and I. Raeburn. Multipliers of imprimitivity bimodules and Morita equivalence of crossed products. Math. Scand., 76:289-309, 1995. CMP 96:02

[ER96] S. Echterhoff and I. Raeburn. The stabilisation trick for coactions. J. Reine Angew. Math., 470:181-215, 1996. CMP 96:07

[GL89] E. Gootman and A. Lazar. Applications of non-commutative duality to crossed product $C^{*}$-algebras determined by an action or coaction. Proc. London Math. Soc., 59:593 624, 1989. MR 91b:46069

[Gre78] P. Green. The local structure of twisted covariance algebras. Acta Math., 140:191-250, 1978. MR 58:12376

[Kal94] S. Kaliszewski. Morita Equivalence Methods for Twisted $C^{*}$-Dynamical Systems. PhD thesis, Dartmouth College, 1994.

[KQ] S. Kaliszewski and J. Quigg. Imprimitivity for $C^{*}$-coactions of non-amenable groups. Math. Proc. Camb. Phil. Soc. to appear.

[Lan95] E. C. Lance. Hilbert $C^{*}-$ Modules. London Math Soc. Lecture Note Series 210. Cambridge University Press, 1995. MR 96k:46100

[Man91] K. Mansfield. Induced representations of crossed products by coactions. J. Funct. Anal., 97:112-161, 1991. MR 92h:46095 
[Ng95] C. K. Ng. Coactions and crossed products of Hopf $C^{*}$-algebras II: Hilbert $C^{*}$-modules. preprint, 1995. CMP 96:09

[Nil96] M. Nilsen. Duality for crossed products of $C^{*}$-algebras by non-amenable groups. preprint, 1996.

[OP86] D. Olesen and G. K. Pedersen. Partially inner $C^{*}$-dynamical systems. J. Funct. Anal., 66:262-281, 1986. MR 87m:46136

[PR89] J. Packer and I. Raeburn. Twisted crossed products of $C^{*}$-algebras. Math. Proc. Camb. Phil. Soc., 106:293-311, 1989. MR 90g:46097

[PR94] J. Phillips and I. Raeburn. Twisted crossed products by coactions. J. Austral. Math. Soc. (Ser. A), 56:320-344, 1994. MR 95e:46079

[QR95] J. C. Quigg and I. Raeburn. Induced $C^{*}$-algebras and Landstad duality for twisted coactions. Trans. Amer. Math. Soc., 347(8):2885-2915, August 1995. MR 95j:46080

[QS92] J. C. Quigg and J. Spielberg. Regularity and hyporegularity in $C^{*}$-dynamical systems. Houston J. Math., 18:139-151, 1992. MR 93c:46122

[Qui95] J. C. Quigg. Full and reduced $C^{*}$-coactions. Math. Proc. Camb. Phil. Soc., 116:435 450, 1995. MR 95g:46126

[Rae81] I. Raeburn. On the Picard group of a continuous trace $C^{*}$-algebra. Trans. Amer. Math. Soc., 263:183-205, 1981. MR 82b:46090

[Rae88] I. Raeburn. Induced $C^{*}$-algebras and a symmetric imprimitivity theorem. Math. Ann., 280:369-387, 1988. MR 90k:46144

[Rae92] I. Raeburn. On crossed products by coactions and their representation theory. Proc. London Math. Soc., 3(64):625-652, 1992. MR 93e:46080

[Rie74] M. A. Rieffel. Induced representations of $C^{*}$-algebras. Adv. Math., 13(2):176-257, 1974. MR 50:5489

Department of Mathematics, University of Newcastle, Newcastle, New South Wales 2308, Australia

E-mail address: kaz@frey.newcastle.edu.au

Department of Mathematics, Arizona State University, Tempe, Arizona 85287

E-mail address: quigg@math.la.asu.edu

Department of Mathematics, University of Newcastle, Newcastle, New South Wales 2308, Australia

E-mail address: iain@frey.newcastle.edu.au 